\title{
The chromospheric component of coronal bright points
}

\section{Coronal and chromospheric responses to magnetic-flux emergence ${ }^{\star}$}

\author{
Maria S. Madjarska ${ }^{1,2}$, Jongchul Chae ${ }^{1}$, Fernando Moreno-Insertis ${ }^{3,4}$, Zhenyong Hou ${ }^{5}$, Daniel Nóbrega-Siverio ${ }^{6,7}$, \\ Hannah Kwak ${ }^{1}$, Klaus Galsgaard ${ }^{8}$, and Kyuhyoun $\mathrm{Cho}^{1}$

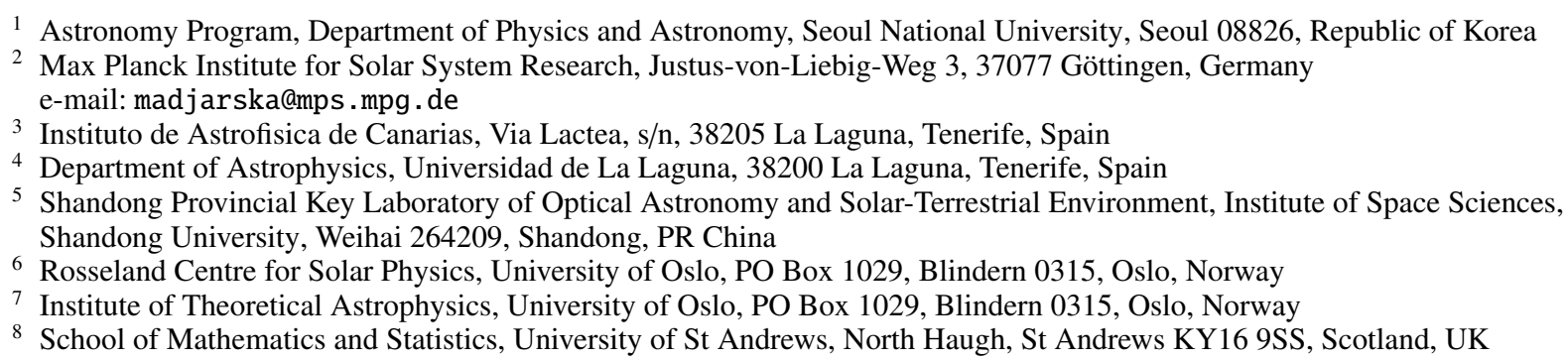

Received 3 September 2020 / Accepted 4 December 2020

\begin{abstract}
Context. We investigate the chromospheric counterpart of small-scale coronal loops constituting a coronal bright point (CBP) and its response to a photospheric magnetic-flux increase accompanied by co-temporal CBP heating.

Aims. The aim of this study is to simultaneously investigate the chromospheric and coronal layers associated with a CBP, and in so doing, provide further understanding on the heating of plasmas confined in small-scale loops.

Methods. We used co-observations from the Atmospheric Imaging Assembly and Helioseismic Magnetic Imager on board the Solar Dynamics Observatory, together with data from the Fast Imaging Solar Spectrograph taken in the H $\alpha$ and Ca II $8542.1 \AA$ lines. We also employed both linear force-free and potential field extrapolation models to investigate the magnetic topology of the CBP loops and the overlying corona, respectively. We used a new multi-layer spectral inversion technique to derive the temporal variations of the temperature of the $\mathrm{H} \alpha$ loops (HLs).

Results. We find that the counterpart of the CBP, as seen at chromospheric temperatures, is composed of a bundle of dark elongated features named in this work $\mathrm{H} \alpha$ loops, which constitute an integral part of the CBP loop magnetic structure. An increase in the photospheric magnetic flux due to flux emergence is accompanied by a rise of the coronal emission of the CBP loops, that is a heating episode. We also observe enhanced chromospheric activity associated with the occurrence of new HLs and mottles. While the coronal emission and magnetic flux increases appear to be co-temporal, the response of the $\mathrm{H} \alpha$ counterpart of the CBP occurs with a small delay of less than $3 \mathrm{~min}$. A sharp temperature increase is found in one of the HLs and in one of the CBP footpoints estimated at $46 \%$ and 55\% with respect to the pre-event values, also starting with a delay of less than 3 min following the coronal heating episode. The low-lying CBP loop structure remains non-potential for the entire observing period. The magnetic topological analysis of the overlying corona reveals the presence of a coronal null point at the beginning and towards the end of the heating episode.

Conclusions. The delay in the response of the chromospheric counterpart of the CBP suggests that the heating may have occurred at coronal heights.
\end{abstract}

Key words. Sun: chromosphere - Sun: corona - Sun: activity - Sun: magnetic fields - methods: observational methods: data analysis

\section{Introduction}

Coronal bright points (CBPs) have been intensively studied for almost five decades. The CBPs are constituted by a set of smallscale coronal loops with an average height of $6500 \mathrm{~km}$ that connect photospheric magnetic-flux concentrations of opposite polarity. As the plasma confined in these loops is heated to a million degrees, they are seen with enhanced emission in the extreme-ultraviolet (EUV) and X-ray wavelengths. The CBPs are found to be uniformly distributed in the quiet Sun and coronal holes and also appear in the vicinity of active regions. For a review of CBPs, see Madjarska (2019).

\footnotetext{
^ Movies are available at https://www . aanda.org
}

The CBP emission throughout EUV and X-ray wavelengths shows conspicuous spatial and temporal variabilities (for a review, see Sect. 5.1 in Madjarska 2019). The first simultaneous observations of CBPs in spectral lines formed at chromospheric, transition region, and coronal temperatures were reported by Habbal \& Withbroe (1981). The study revealed that the EUV emission from CBPs varies dynamically on short timescales. The emission enhancements in coronal lines were found to have a counter-response in chromospheric and transition-region lines. The analysed spectroheliograms were taken with the Harvard EUV experiment on Skylab/ATM at a 5" spatial resolution and $5.5 \mathrm{~min}$ cadence in the C II $1335 \AA$, Ly- $\alpha 1216 \AA$, O IV $554 \AA$, O VI $1032 \AA$, C III $977 \AA$, and MgX $625 \AA$ lines. 
From observations taken near to the solar limb, it was found that the increase of the coronal emission in CBPs precedes the lower temperature emission. The authors therefore suggested that the heating possibly occurs at coronal heights and is carried down to the chromosphere by thermal conduction. This is the first and only study that has investigated the heating of CBP plasmas by exploring the temporal response in spectral lines with various formation temperatures. From the analysis of images taken with the SECCHI Extreme Ultraviolet Imager (EUVI) on board the STEREO (Solar TErrestrial RElations Observatory) twin spacecrafts in four passbands at $171 \AA, 195 \AA, 284 \AA$, and $304 \AA$, Kwon et al. (2012) concluded that CBPs are composed of hot loops $(\log T(\mathrm{~K}) \sim 6.2)$ overlying cooler loops $(\log T(\mathrm{~K}) \sim 6.0)$ with cool legs $(\log T(\mathrm{~K}) \sim 4.9)$.

The Doppler velocities in CBPs were reviewed in detail by Madjarska (2019). In this work, we only recapture details that are relevant to the present study. Madjarska et al. (2003) studied a single case of a $\mathrm{CBP}$ in a coronal hole using rasterimage observations in the S VI $933.40 \AA$ line $\left(T \approx 2.0 \times 10^{5} \mathrm{~K}\right.$ maximum formation temperature) taken with the Solar Ultraviolet Measurements of Emitted Radiation (SUMER) spectrometer on board the Solar and Heliospheric Observatory (SoHO). The locations above the bipole magnetic-flux concentrations were associated with red-shifted emission (i.e. the footpoints of the CBP loops), while blue-shifted emission was detected in the top or centre of the CBP loops. The blue-shifted signal was interpreted as coming from the underlying coronal hole rather than the CBP loops. We should not exclude the possibility that the emission from low-lying transition-region loops has also contributed to the blue-shifted emission. Because of resolution limitations, the SUMER spectrometer could not resolve individual small-scale loops, which is now possible with the Interface Region Imaging Spectrograph (IRIS). Kayshap \& Dwivedi (2017) analysed two CBPs in data obtained with IRIS as well as with the Atmospheric Imaging Assembly (AIA) and Helioseismic Magnetic Imager (HMI) on board the Solar Dynamics Observatory (SDO). The CBP loops were also seen in the IRIS slit-jaw images (SJIs) taken in the Mg II k $2796 \AA$ (chromosphere) and C II $1330 \AA$ (low transition region) passbands. Small-scale loops can be distinguished in the SJIC II channel connecting bright-point-like sources that are the locations of the magnetic-flux concentrations. As in Madjarska et al. (2003), the study found red-shifted emission in the transition-region Si IV $1393.75 \AA\left(8.0 \times 10^{4} \mathrm{~K}\right)$ line in the range $5-30 \mathrm{~km} \mathrm{~s}^{-1}$, $\sim 5-20 \mathrm{~km} \mathrm{~s}^{-1}$ in C II $1394.76 \AA\left(2.5 \times 10^{4} \mathrm{~K}\right)$, and $\sim 10 \mathrm{~km} \mathrm{~s}^{-1}$ in O IV $1401.16 \AA\left(1.6 \times 10^{5} \mathrm{~K}\right)$ at the two legs or footpoints of the CBP loops. Blue-shifted emission was recorded in the centre of the CBP. However, the background emission from the coronal hole, as in the case studied by Madjarska et al. (2003), may also have contributed to this emission. In coronal lines, Xia et al. (2003) reported blue-shifted emission from a CBP in the range $3-7 \mathrm{~km} \mathrm{~s}^{-1}$ in Ne VIII $770.42 \AA\left(6.3 \times 10^{5} \mathrm{~K}\right)$ without indication of the site of origin, while Tian et al. (2008) obtained Doppler shifts close to zero in the same line.

The chromospheric counterpart of CBPs has been poorly reported. An example of a study in the chromosphere of a $\mathrm{CBP}$ is the work by Contarino et al. (2009). An arch filament system (AFS) associated with a bipolar region seen in Ca II 8542.1 data taken with the Interferometric Bidimensional Spectrometer (IBIS) on the Dunn Solar Telescope (DST) of the United States National Solar Observatory at Sac Peak was analysed. The AFS was found to connect a bipolar region and to have a counterpart at coronal temperatures in images taken with the
Extreme ultraviolet Imaging Telescope (EIT) on board SoHO. In view of the statements by the authors, we can conclude that this region represents a CBP. The CBP lifetime was $\sim 3.5$ days. The CBP formed following a bipole flux emergence. The magnetic fragments evolved through various processes including divergence, convergence, and cancellation in the latest stage of the bipole lifetime. The velocities were derived from the shift of the Ca II line centroid and cloud model flows (see the paper for details on the methodology). While the velocities obtained from either method are similar at the loop top, these velocities are different in the loop footpoints. The study reports that the lineof-sight (LOS) velocities are similar with values that are greater by a factor 3-4 for the Doppler shift method. In summary, redshifted emission is known to dominate at the footpoints or legs of CBP loops. Blue-shifted emission is observed at the centre or top of the loops at transition-region temperatures, but the contribution from the background emission of the coronal hole can be important, especially in spectral lines formed at higher transition region and coronal temperatures.

Magnetic flux emergence from the convection zone into the overlying solar atmosphere is an essential physical process that has a fundamental impact in the activity of the solar atmosphere on a wide range of length scales (for a review see Cheung \& Isobe 2014). Magnetic-flux emergence in the quiet Sun causes the formation of CBPs in around $50 \%$ of all observed cases (e.g., Mou et al. 2018, and references therein). Typically $30 \mathrm{~min}$ to $60 \mathrm{~min}$ after a visual detection of the emerging bipolar flux in the photosphere, the first CBP coronal loops can be detected in AIA 193, often with projected size as small as $5^{\prime \prime}$. In active regions magnetic flux emergence is linked to the formation of various eruptive phenomena, including Ellermann bombs (e.g., Hansteen et al. 2019, and references therein), surges (e.g., Nóbrega-Siverio et al. 2017, and references therein), filament eruptions, and coronal mass ejections (e.g., Dacie et al. 2018, and references therein).

In the present paper we investigate the temporal evolution of a CBP as seen in the AIA 193 and $304 \AA$ channels of SDO in parallel with the simultaneous evolution in morphology, dynamics, and temperature obtained from chromospheric lines ( $\mathrm{H} \alpha$ and Ca II 8542.1 A) based on data taken with the Fast Imaging Solar Spectrograph (FISS). A CBP brightness increase is observed in the $193 \AA$ channel of SDO/AIA and is associated with a photospheric magnetic-flux increase recorded in LOS SDO/HMI magnetograms. For the first time, magnetic field lines obtained from a linear force-free field (LFFF) extrapolation model are matched with CBP loops seen in images taken with the AIA $193 \AA$ filter and their parameters are derived. The paper is organized as follows. Section 2 reports on the analysed observational material and the methodology used to derive various physical parameters. The results are given in Sect. 3. The discussion is presented in Sect. 4 and the summary and conclusions are in Sect. 5.

\section{Observational material and methodology}

The chromospheric observations were taken with FISS at the Big Bear Solar Observatory on 2017 June 14. The FISS is a dual-band échelle spectrograph recording simultaneously the $\mathrm{H} \alpha$ and the Ca II 8542.1 $\AA$ lines (Chae et al. 2013). The images are produced in a raster mode with a $0.16^{\prime \prime}$ wide slit. The data are obtained using an adaptive optics system. The spectral sampling is $0.019 \AA$ for the $\mathrm{H} \alpha$ line and $0.026 \AA$ for the Ca II line. The raster cadence is $\sim 27 \mathrm{~s}$ with a raster time step of $0.13 \mathrm{~s}$. The data were taken in the quiet Sun. The images in the far wings of $\mathrm{H} \alpha$ 
and Ca II were used to align with the HMI magnetograms. The field of view (FOV) is $24^{\prime \prime} \times 40^{\prime \prime}(1.74 \mathrm{Mm} \times 29 \mathrm{Mm})$ and it is tilted with respect to the north-south direction. Only observations taken between 17:52:03 UT and 18:49:33 UT were used for the analysis as they had the best quality.

To obtain the Doppler velocities we used the lambdameter method that is illustrated and described in Chae et al. (2014, and references therein). The obtained Doppler velocities are not absolute values as they are obtained with respect to a reference spectrum from a region away from the CBP (see Sect. 3 for more details). Nevertheless the Doppler shifts give a correct image of the velocity pattern and its evolution in time. The hydrogen temperature at the chromospheric heights under the CBP is obtained from a new multi-layer spectral inversion technique developed by Chae et al. (2020).

To investigate the coronal part of the CBP, we analysed imaging data taken by AIA (Lemen et al. 2012) on board SDO (Pesnell et al. 2012). We used AIA data taken in the $193 \AA$ (hereafter AIA 193) and $304 \AA$ (hereafter AIA 304) channels. The AIA 193 channel is dominated by Fe XII lines $(\log T(\mathrm{~K}) \sim 6.2)$. The AIA 304 channel is dominated by the two He II lines at 303.786 and $303.781 \AA(\log T(\mathrm{~K}) \sim 4.7)$, but this channel also has a contribution from the coronal Si XI at $303.33 \AA$ $(\log T(\mathrm{~K}) \sim 6.2$ ) (O'Dwyer et al. 2010). The formation of the He II lines is complex (Andretta et al. 2012; Golding et al. 2017) and the AIA 304 channel is known to predominantly show the footpoints of coronal loops as well as in CBP loops. The contribution by $\mathrm{SiXI}$ at $303.33 \AA$ is estimated to be at the $11 \%$ level in active regions (O'Dwyer et al. 2010) implying that the AIA 304 channel may also show a contribution from the coronal emission in CBPs. Chromospheric low-temperature features appear dark in these channels (but also in other coronal channels). This is caused by the extinction of photons emitted from the hot coronal plasma in overlying neutral H I or He I atoms in cool plasma (Rutten 1999). The AIA EUV data have a $12 \mathrm{~s}$ cadence and $0.6^{\prime \prime} \times 0.6^{\prime \prime}$ pixel size.

The HMI LOS magnetograms (Scherrer et al. 2012) used in this study have either a $45 \mathrm{~s}$ or a $720 \mathrm{~s}$ cadence and $0.5^{\prime \prime} \times 0.5^{\prime \prime}$ pixel size. The magnetograms with $720 \mathrm{~s}$ cadence were used for the LFFF extrapolation because of their higher signal-to-noise ratio. The $45 \mathrm{~s}$ cadence data were used for the co-temporal FISS study. The magnetic field data were corrected for the projection effect. The AIA $1600 \AA$ was used to align the HMI data with those from AIA $193 \AA$. The precision of the alignment between the different instruments is $\sim 1^{\prime \prime}$. To produce the curves of the positive and negative fluxes over the CBP full lifetime we used a 5 min data-cadence sequence. All images in this work were rescaled to the FISS pixel size, which is $0.16^{\prime \prime}$. The AIA 193, $\mathrm{H} \alpha$, and Ca II curves were smoothed using a sevenpoint window, while for the HMI data, a four-point window was applied.

We investigated the topology of the magnetic field, both of the low-lying loops and of the overlying coronal structure. To obtain the magnetic structure of the CBP loops, we first processed the AIA 193 images using the multi-scale Gaussian normalization (MGN) code developed by Morgan \& Druckmüller (2014). Next, we applied a LFFF extrapolation model using the LOS magnetic-field measurements from HMI. A low average plasma- $\beta$ allowed us to assume that to the lowest order the magnetic field is force-free; that is the current density is aligned with the magnetic field. Details concerning the methodology can be found in Wiegelmann \& Neukirch (2002) and Wiegelmann et al. (2010). The LFFF contains the force-free parameter $\alpha\left(\mathrm{m}^{-1}\right)$.
In this work, the dimensionless quantity $\alpha \mathrm{L}$ is used, where $L$ is the harmonic mean of the sides, $L_{x}$ and $L_{y}$, of the magnetogram (i.e. $\left.1 / L^{2}=1 / 2\left(1 / L_{x}{ }^{2}+1 / L_{y}{ }^{2}\right)\right)$. We carried our computations with $\alpha \mathrm{L}$ values in the range from -4.0 to 4.0 , with a step of 0.5 . The MGN image processing helps to enhance the CBP loops so that a visual selection could be made along their entire length. Then, a fit was attempted between the LFFF extrapolation and the visual identification from the MGN image processing. The least-squares method was used to find the field line, which has the minimum distance between the interpolated field lines and the loop visually identified by us.

Additionally, a potential field extrapolation model was applied to the magnetograms taken at 17:21 UT, 18:10 UT, 18:15 UT, and 18:25 UT, to investigate the magnetic topology of the overlying corona and to search for the presence of magnetic null points. Details of the extrapolation methodology are given in Sect. 2.2 of Galsgaard et al. (2017), while the methodology of the magnetic topological analysis is described in Sect. 2.3 of the same paper.

\section{Results}

\subsection{CBP formation and evolution}

In the search for suitable observations we were very fortunate to discover that the highest quality FISS data in the quiet Sun are co-spatial and co-temporal with a CBP despite the FISS small FOV of $24^{\prime \prime} \times 40^{\prime \prime}$. The initial data analysis was even more encouraging as it showed that during the one hour of the highest-quality observations, the CBP had an episode of intensity increase recorded in the AIA 304 and 193 channels that was linked to a rise of the photospheric magnetic flux in the footpoints of the CBP loops.

The CBP was formed from bipolar flux emergence, which is typical for at least $50 \%$ of the CBPs (Mou et al. 2018). The emergence started at $\sim 13: 35$ UT on 2017 June 13 (Fig. 1). The first signature of the CBP in AIA 193 is at 14:44 UT on June 13 and the last time a trace of the CBP is visually detectable in this channel is on June 14 at 23:00 UT, which gives a lifetime of $\sim 33 \mathrm{~h}$. The CBP positive polarity fully vanishes at 02:00 UT on June 15, while a patch of the negative flux remains. Figure 1 (top row images) and Fig. 2 (top row images) contain the HMI magnetograms, which clearly show the opposite polarities where some of the CBP loops are rooted (see Sect. 3.4 for details). The general evolution of the photospheric magnetic flux follows a typical evolutionary pattern seen in CBPs forming from flux emergence (e.g., Mou et al. 2018). The flux emergence proceeds with a continuously diverging bipole until the two polarities reach a certain distance, while the CBP attains its largest size and brightness. Thereafter, a magnetic flux convergence process follows until the full disappearance of one of the polarities (the positive one). Occasionally flux coalescence and cancellation are observed. The cancellation is most prominent towards the end of the lifetime of the CBP. During the magnetic flux emergence the positive flux approaches a pre-existing negative flux, which leads to micro-flaring and to a mini-ejection (this event will be the subject of investigation in a follow-up study). During the specific period of the FISS observations, a displacement of $\sim 0.27 \pm 0.03 \mathrm{~km} \mathrm{~s}^{-1}$ of the positive flux was approximately estimated while the negative flux remained almost at the same location.

We paid attention to the full lifetime evolution of the coronal intensity, as recorded in AIA 193, and to the bipolar magnetic flux. As in all emergence cases shown in Mou et al. (2018), 

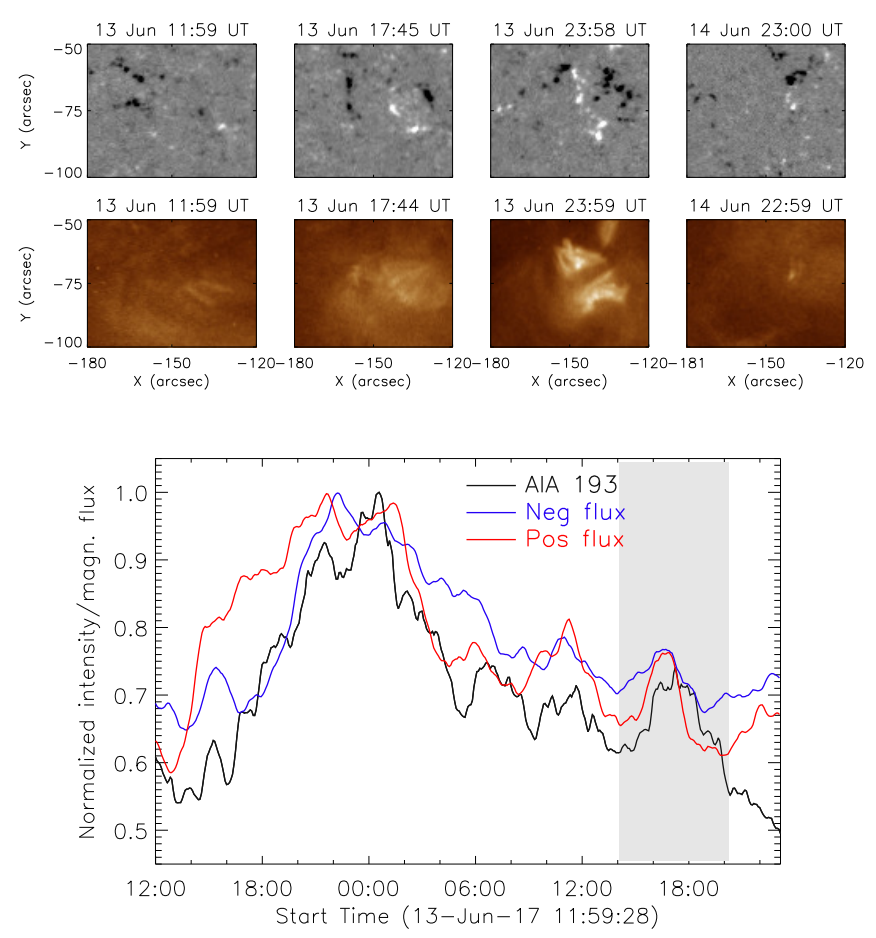

Fig. 1. Temporal variation of the magnetic flux and AIA 193 intensity of the CBP along its full lifetime. Images at the top: HMI saturated at $\pm 100 \mathrm{G}$ (top row) and AIA 193 (bottom row). Bottom panel: AIA 193 intensity and LOS magnetic flux of the positive and negative polarities. Each curve is normalized to its maximum. The grey area highlights a period of magnetic flux and coronal emission increase, part of which is covered by the FISS data and is analysed here in detail.

there is a strong correlation between the temporal evolution of the coronal intensity recorded in AIA 193 and the photospheric magnetic flux. In Fig. 1 we show the temporal variations of the normalized AIA 193 intensity of the CBP over its lifetime, and the normalized total positive and negative magnetic fluxes. In the top panel of Fig. 1 we show two rows (top - HMI, bottom - AIA 193) consisting of four images each that display the time evolution of the CBP, including its appearance, the phase of maximum size and intensity, and its disappearance. The window is slightly offset from the centre of the CBP to catch the CBP loops that extend to the east, since the CBP is not observed in the disc centre. The curves in the bottom panel of Fig. 1 were produced using the $5 \mathrm{~min}$ cadence data and from a FOV slightly larger than the one shown in Fig. 2 in order to enclose the CBP while reaching its maximum size earlier in its lifetime ( 00:00 UT on June 13). A heating event occurred between $\sim 18: 07$ UT and $\sim 18: 33$ UT on June 14, that is towards the end of the CBP lifetime, which we analyse in the following sections. The curves in Fig. 1 reveal that the period of time covered by the FISS data is part of a longer lasting heating event following a magnetic-flux increase that started around 14:00 UT on June 14 (shown with a grey background). As is generally the case for the whole evolution of the CBP, there is a strong correlation between the magnetic flux increase (emergence) and enhancement of the coronal emission. The heating event lasted a bit less than $3 \mathrm{~h}$. For the full lifetime of the CBP, the linear Pearson correlation coefficient for the positive flux and the AIA 193 intensity is 0.75 , while for the negative flux it is 0.88 . As not all the flux in the FOV is involved in the CBP magnetic configuration, the correlation coefficient is at least 0.9 .

\subsection{Chromospheric and coronal morphology of the CPB}

The $\mathrm{H} \alpha$ line-core intensity images in (the third row of Fig. 2) show the CBP chromospheric morphology, which can simply be described as a bundle of slightly curved loops (only the southern end is sigmoid shaped) that connect a bipolar region. The $\mathrm{H} \alpha$ line-core intensity was derived from the lambdameter method (see Sect. 2). The loops are seen as elongated dark features that lie along the path between the magnetic polarities of opposite flux. In Fig. 3 we show images that were produced by summing the intensity flux in the wings of $\mathrm{H} \alpha$ at $\pm 0.45 \AA$, where the structure of the evolving loops of the CBP can best be followed. Hereafter we use the term $\mathrm{H} \alpha$ loops (HLs) of the CBP, as these features are certainly part of the whole CBP magneticplasma complex. Often studies refer to these phenomena in the quiet Sun as arch filaments (e.g., Contarino et al. 2009, and references therein) or fibrils (e.g., Leenaarts \& Carlsson 2012, and references therein), while in active regions they are named fibrils (e.g., Cauzzi et al. 2008; Tsiropoula et al. 2012).

The second and third rows of panels in Fig. 2 allow us to compare the line-core intensity of the CaII line (second row) with those of $\mathrm{H} \alpha$ just mentioned. The footpoints of the HLs are rooted in the magnetic-flux concentrations of opposite polarity and appear as bright features in both the $\mathrm{H} \alpha$ and the $\mathrm{Ca}$ II images as typically seen above magnetic-field concentrations. In the line wings the brightenings have sharp edges while in the line centres they appear larger and more diffuse at the edges because of the lateral divergence of the magnetic field with height. In addition to the HLs, we observe dynamically evolving absorption features that both rise and fall back on short timescales (a few minutes). These features are known as mottles (for details on mottles see Tsiropoula et al. 2012, and references therein) and are best seen in the wings of the $\mathrm{H} \alpha$ line (see the animation in Fig. A.1) emanating from the magnetic concentrations, which also host the footpoints of the CBP loops (e.g., Contarino et al. 2009). Mottles are known to propagate both along closed and open magnetic field lines (Tsiropoula et al. 2012). We also observe up- and downflows in the legs of the HLs. These flows possibly would have been identified as mottles by a mottle-dedicated study, but hereafter we consider them as a separate feature because of their association with the CBP magnetic structure. A closer look at the footpoints of the CBP loops in the AIA 193 images also reveals these dark (absorption) dynamically evolving features (see the animation in Fig. A.1). The combination of $\mathrm{H} \alpha$ and AIA 193 images clearly demonstrates that mottles and cool plasma upflows in the legs of the CBP loops are responsible for the darkening in the legs or footpoints and the adjacent areas of the CBP loops. This causes for instance the absence of coronal emission above the northern CBP footpoint at the location of the negative polarity creating the illusion of the coronal emission from the CBP to be misaligned with respect to the magnetic polarities. In the south footpoint (positive magnetic polarity) the loops are sigmoidal and therefore do not create such an obscuration. Often the mottle or cool-plasma-flow evolution also creates small-scale temporal and spatial variations of the coronal intensity. In reality, these are the absorption features that evolve dynamically along the CBP loop legs and even rise up along the loops. This can be followed in the provided animation in Fig. A.1. In the AIA 304 images in Fig. 2 we can clearly distinguish the bright footpoints of the CBP as well as the so-called legs (the low part of the CBP loops), which is common when CBPs are observed at transition-region temperatures (for details see Madjarska 2019). The CBP loops also become visible in this channel during the analysed observing period. We come back to 


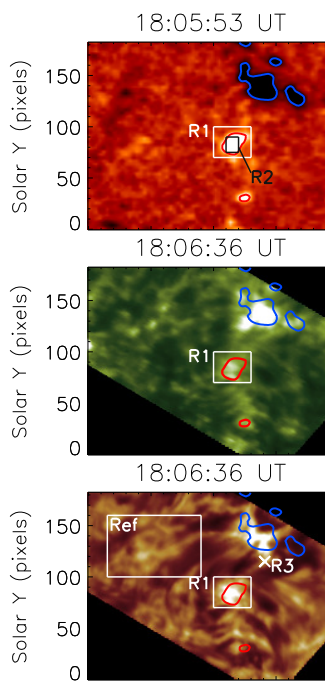

18:06:29 UT

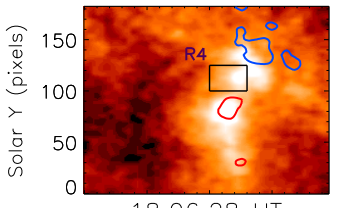

18:06:28 UT

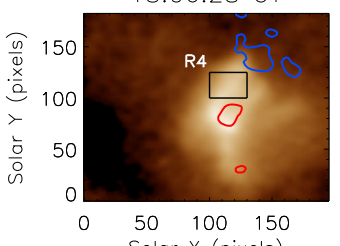

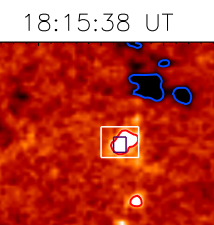

18:15:53 UT

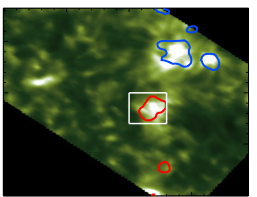

18:15:53 UT

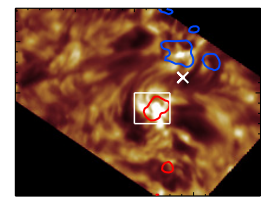

18:15:53 UT

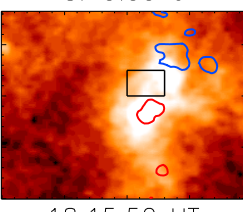

18:15:52 UT

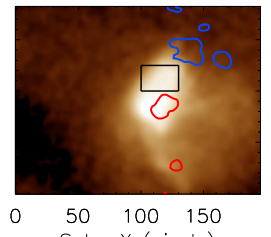

18:24:38 UT

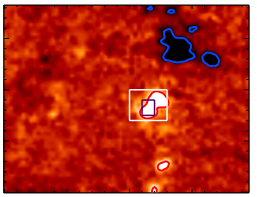

18:24:43 UT

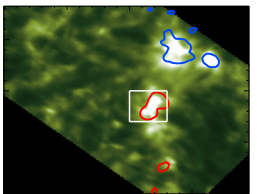

$18: 24: 43$ UT

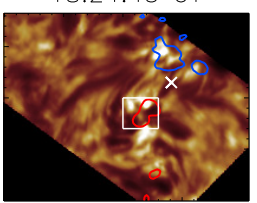

18:24:41 UT

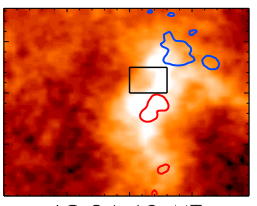

$18: 24: 40$ UT

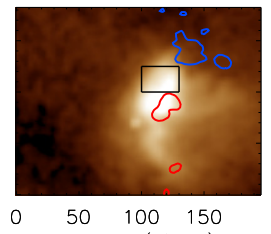

18:33:38 UT

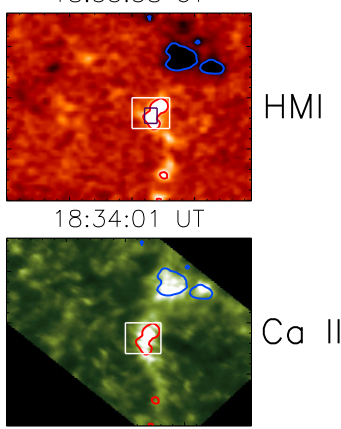

$18: 34: 01$ UT

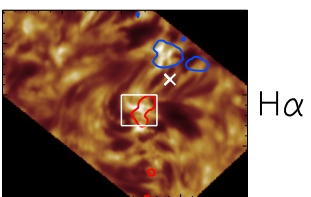

8:33:53 UT

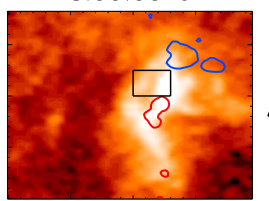

AIA 304

18:33:52 UT

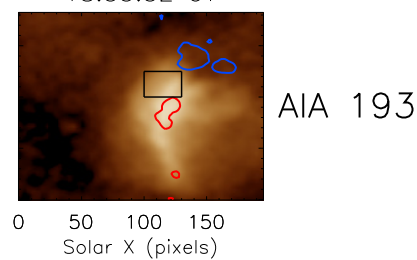

Fig. 2. Multi-instrument images of the CBP. Top row: HMI line of sight magnetograms scaled from -50 to $50 \mathrm{G}$ overplotted with a larger square noted as region of interest \#1 (R1, also on the $\mathrm{H} \alpha$ and Ca II images), which outlines the area from which the temporal variations of various parameters in the south footpoint of the CBP were obtained. The smaller square denotes (R2) the area from which the hydrogen temperature was determined in Fig. 7. Second row: Ca II line-core intensity images overplotted with the $\pm 50 \mathrm{G}$ magnetic-field contours. Third row: H $\alpha$ line-core intensity images with the same contours. The large square on the $\mathrm{H} \alpha$ image in the first column (Ref) is the area from which the reference $\mathrm{H} \alpha$ profile used to derive the Doppler velocities was obtained. The cross sign denotes the centre of a small area (R3) from which the temporal variations in an $\mathrm{H} \alpha$ loop were produced in Figs. 5 and 7. Fourth and fifth rows: AIA 304 and AIA 193 images overplotted with the $\pm 50 \mathrm{G}$ magnetic-field contours and a square (R4) that outlines the area from which the light curve in the CBP loops was produced for Figs. 5-7. The pixel size is 0.16", that is the size of the images is $29^{\prime \prime} \times 31^{\prime \prime}$.

this later in the next section. The north CBP footpoint is also obscured by mottles as in the coronal AIA 193 channel. Chromospheric features such as filaments and mottles or spicules are usually seen in absorption when observed on the disc in AIA 304 (e.g., Madjarska et al. 2020, and references therein).

While the HLs evolve more dynamically, the coronal loops show little change (see the animation in Fig. A.1). This, however, may be due to the different spatial resolution of the two instruments, which is $0.16^{\prime \prime} \mathrm{px}^{-1}$ for the FISS chromospheric data and $0.6^{\prime \prime} \mathrm{px}^{-1}$ for the AIA data. The cross-section of several HLs were measured and an average width of $\sim 0.41^{\prime \prime}$, with a minimum size of $0.28^{\prime \prime}$ and maximum of $0.56^{\prime \prime}$, were obtained. These were determined from the FWHM of their absorption profile cross-section.

The top and bottom panels of Fig. 4 show the Doppler-shift images in the $\mathrm{H} \alpha$ and Ca II lines, respectively, obtained through the lambdameter method. We note that the Doppler shifts are calculated relative to an average profile taken from the area denoted with the larger square on the first panel of the $\mathrm{H} \alpha$ images in Fig. 2. Thus, the Doppler velocities are only indicative of the flow patterns. Corresponding animations of the Doppler-shift $\mathrm{H} \alpha$ and $\mathrm{Ca}$ II temporal sequences are also provided in Figs. A.1 and A.2, respectively. Despite the obviously complex structure of this region, the Doppler maps clearly show the pattern of the HLs that appear with elongated blue- or red-shifted emission. Predominant downflows are observed in the legs of the CBP loops, but upflows with an elongated shape associated with the CBP structure are also present. Mottles are also easy to detect propagating in all directions forming the so-called rosettes (see Sect. 4.2.1 in Tsiropoula et al. 2012).

\subsection{Chromospheric and coronal response to magnetic flux increase}

\subsubsection{Intensity and Doppler-shift temporal evolution}

Although the CBP appears relatively unchangeable visually in the AIA 193 channel during the FISS observing period, a shortlived brightness increase made us inspect the coronal intensity in more detail. We derived the intensity at the chromospheric $(\mathrm{H} \alpha)$, transition-region (AIA 304), and coronal (AIA 193) temperatures, magnetic flux, Doppler-shift, and hydrogen-temperature temporal variations from selected regions of interest $(\mathrm{R})$ of the CBP including the south footpoint of the CBP (denoted as R1 

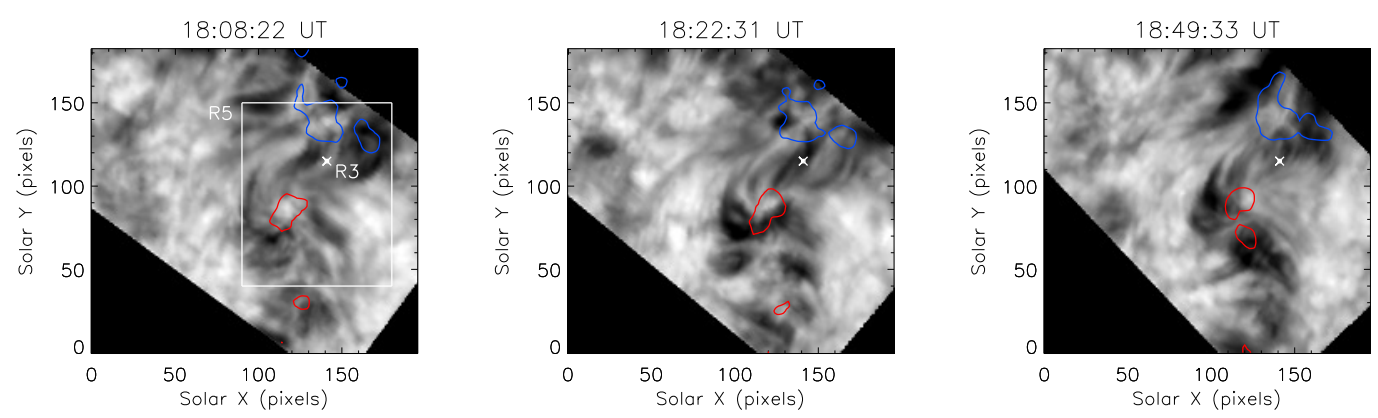

Fig. 3. $\mathrm{H} \alpha$ images before (left), during (middle) and after (right) the heating episode with overplotted the $\pm 50 \mathrm{G}$ magnetic-field contours (red positive polarity, blue - negative). The images are produced by summing the $\mathrm{H} \alpha$ intensity in the blue and red wings of $\mathrm{H} \alpha$ at $\pm 0.45 \AA$. The large square denoted as R5 in the left panel is where the temporal variations from the whole CBP shown in Figs. 5 and 7 were obtained. A cross denotes R3, from which the temporal variations in one of the HLs were produced in Figs. 5 and 7. The pixel size is $0.16^{\prime \prime}$.
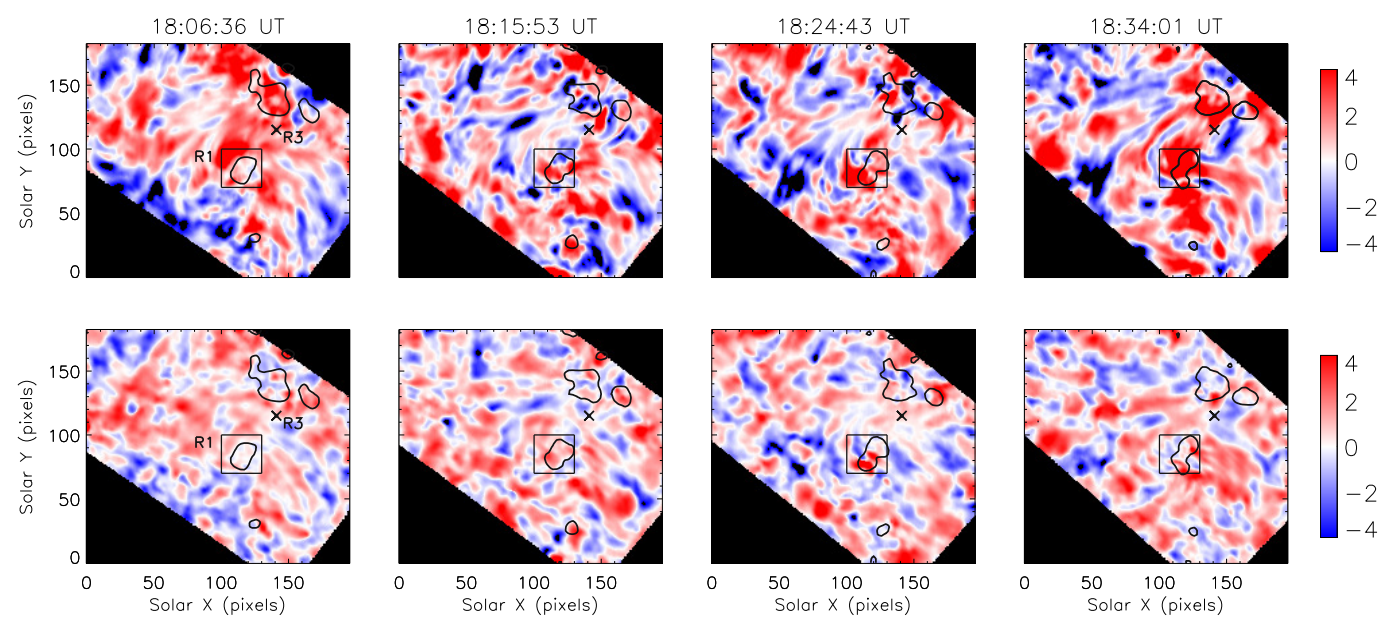

Fig. 4. $\mathrm{H} \alpha$ (top row) and Ca II (bottom row) Doppler velocity images at the corresponding times as in Fig. 2. The square indicates where the Doppler-shift temporal variation in the south footpoint was obtained. The cross denotes the centre of the small area from which the Doppler-shift temporal variation in the $\mathrm{H} \alpha$ loop was obtained. The pixel size is $0.16^{\prime \prime}$.

and R2 in Fig. 2), an individual HL (R3), the CBP loops (R4), and the whole CBP (R5 in Fig. 3).

The top panel of Fig. 5 shows the total magnetic flux variations taken from the region of the positive flux outlined with a larger square (R1) in the top row of Fig. 2. We chose only the positive magnetic flux associated with the CBP because it is centrally located in the FISS FOV and almost exclusively relates to the CBP, that is it provides a less "contaminated" signal. The negative magnetic flux at the location of the CBP north footpoint is more dispersed and has more complex connectivities that are not related to the CBP. The top panel of Fig. 5 also presents the AIA 193 averaged intensity variations taken from an area of the CBP coronal loops (R4). We did not select the entire CBP because the AIA 193 channel is also contaminated by transitionregion emission. The inclusion of the $\mathrm{CBP}$ footpoints, and, especially, the south footpoint of the CBP that is not obscured by mottles and cool-plasma upflows and strongly emit at low temperatures (O'Dwyer et al. 2010), may contaminate the extracted coronal emission. We also present the AIA 304 temporal variations taken from the south footpoint (R1).

Starting at $\sim$ 18:07 UT, a magnetic-flux increase (Fig. 5, top panel) is detected reaching a maximum after $\sim 8$ min. A second but weaker peak follows $\sim 10$ min later. For the first peak, the magnetic flux increase is $\sim 11 \%$ with respect to the pre-event value $\left(\sim 6.0 \times 10^{18} \mathrm{Mx}\right)$ which corresponds to a total magnetic-flux increase taken from an area of $31 \times 31 \mathrm{px}^{2}$, of $\sim 6.7 \times 10^{17} \mathrm{Mx}$ for the first peak, and $\sim 6.4 \times 10^{17} \mathrm{Mx}$ for the second at $\sim 18: 25$ UT. The magnetic flux has been identified as a magnetic-flux emergence event through the co-temporal increase of the negative flux where the HLs are rooted. At $\sim 18: 30$ UT the magnetic flux drops to the values it had prior to the event. A co-temporal coronal intensity rise is detected in the coronal loops registered in AIA 193 starting at 18:07 UT. Surprisingly, the intensity increase in the AIA 304 channel clearly precedes both the magnetic flux and the AIA 193 intensity by a bit more than $1 \mathrm{~min}$. The emission also persists a minute longer after the AIA 193 in the coronal loops. The most plausible explanation is that AIA 304 depicts the effects of the magnetic flux emergence earlier than the HMI and only when the magnetic flux reaches above certain threshold of detection it is recorded by HMI. The same behaviour of the AIA 304 emission is seen around 18:00 UT where a small increase of the magnetic flux again lags behind the transition region emission. This explains also the surprising lack of delay between the magnetic flux emergence and the coronal response, which is commonly observed during CBP formation (for details see Table 1, last column in Mou et al. 2018). Follow-up studies hopefully will shed more light on the behaviour of the AIA 304 emission in CBPs.

We should note that the HMI cadence is $45 \mathrm{~s}$, and, therefore, the data are not fully co-temporal. We prepared image sequences of the AIA 193, HMI, and FISS data, which are as close to each other in time as possible; see the animation in Fig. A.1. The clearly identifiable period of a coronal-intensity increase is roughly marked by two vertical lines (added to all curve 

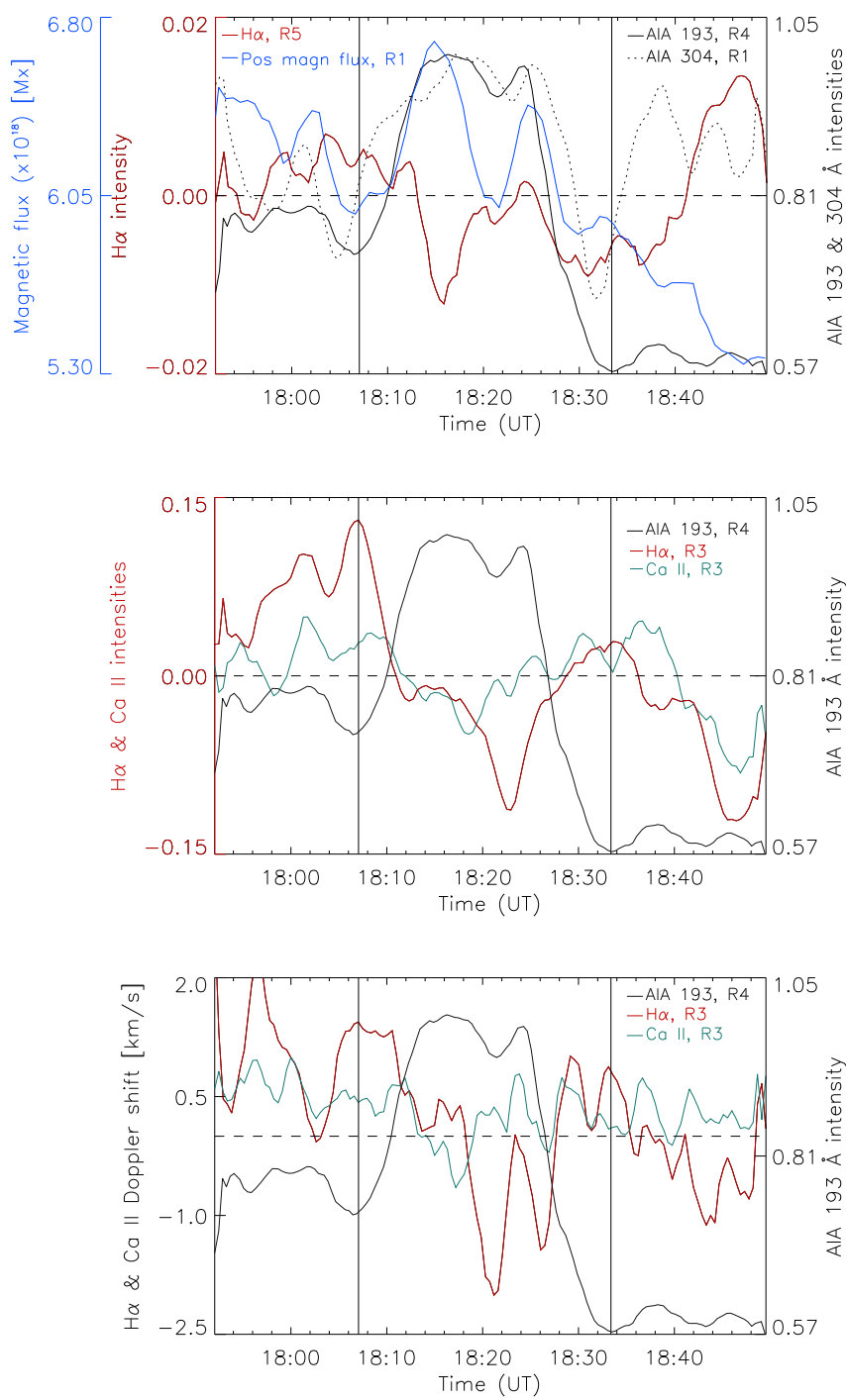

Fig. 5. Temporal variations of the coronal, transition-region, and chromospheric emission, $\mathrm{Ca}$ II and $\mathrm{H} \alpha$ Doppler shift, and positive magnetic flux of the CBP. Top panel: total positive magnetic flux and AIA 304 normalized intensity taken from R1 shown in Fig. 2, AIA 193 normalized intensity produced from R4 shown on the AIA 193 in Fig. 2, and the $\mathrm{H} \alpha$ intensity produced from the sum of the intensity in the blue and red wing at $\pm 45 \AA$ averaged from R5. Middle panel: Ca II and $\mathrm{H} \alpha$ linecore intensities produced from one of the HLs, R3. Bottom panel: Ca II and $\mathrm{H} \alpha$ Doppler-shift variations in R3 as in the middle panel. The horizontal dashed line indicates the zero Doppler-shift value. The AIA 193 intensity temporal variations (R4) are shown for reference in both the middle and bottom panels.

figures in this paper) and has been determined from the coronal (AIA 193) intensity flux variations of the CBP. The AIA 193 intensity rise is approximately $35 \%$ with respect to the preevent value (at $\sim 18: 07$ UT). The maximum intensity is reached in $\sim 9 \mathrm{~min}$ at $\sim 18: 16 \mathrm{UT}$ and it remains relatively constant with a small dip before a second increase apparently related to the second magnetic-flux intensification. We therefore conclude that an enhancement of the magnetic flux due to magnetic-flux emergence observed at photospheric level is associated with a heating of the CBP loop plasma at coronal temperatures. Checking with the long-term evolutionary history of this CBP (see Fig. 1), we see that, after the end of this heating episode, the CBP continued its evolution by becoming smaller and fainter and disappearing some $4 \mathrm{~h}$ later.
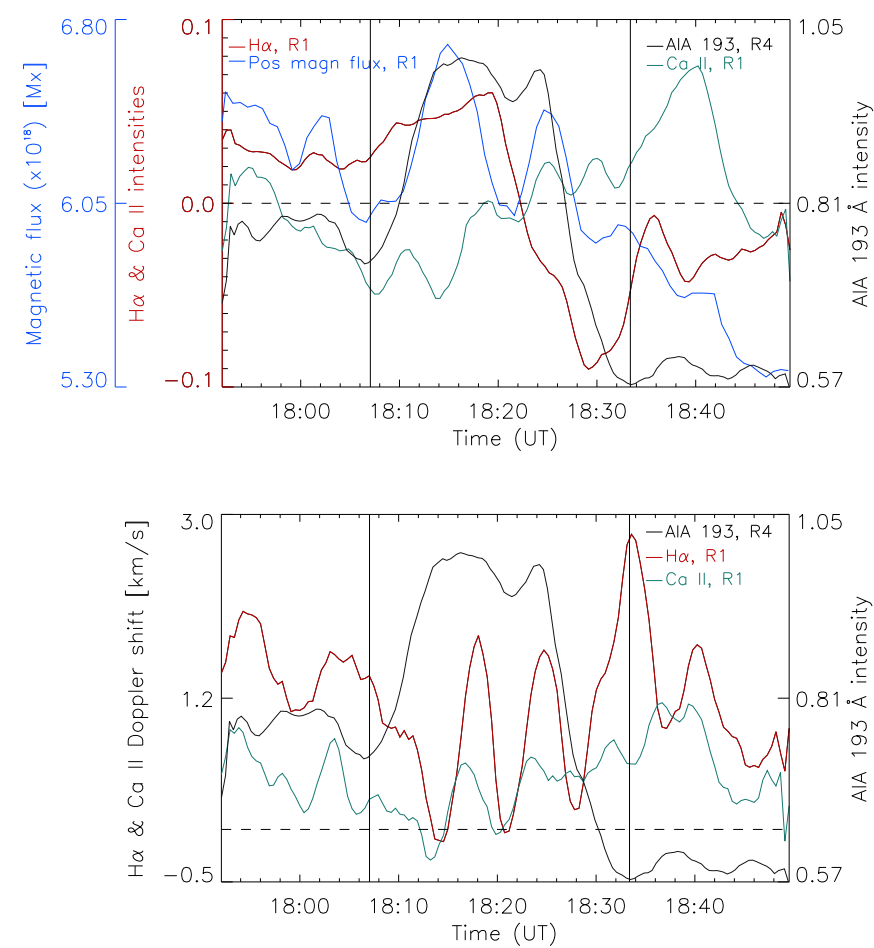

Fig. 6. Temporal variations of the $\mathrm{Ca}$ II and $\mathrm{H} \alpha$ line-core intensities and $\mathrm{Ca}$ II and $\mathrm{H} \alpha$ Doppler shift produced from the south footpoint of the CBP. Top: total positive magnetic flux, $\mathrm{Ca}$ II and $\mathrm{H} \alpha$ line-core intensities taken from R1 together with the AIA 193 intensity from R4. Bottom: $\mathrm{Ca}$ II and $\mathrm{H} \alpha$ Doppler-shift taken from R1, and the AIA 193 intensity from R4. The horizontal dashed line indicates the zero Doppler-shift value.

The Ca II line-core intensity in the HLs and south footpoint varies differently from the $\mathrm{H} \alpha$ line-core intensity (for details see Figs. A.1 and A.2) most likely for two reasons. The HL are optically thin in the Ca II line so that the behaviour of the Ca II light curve is not comparable to the $\mathrm{H} \alpha$ light curve. Another reason is that the Ca II light curve is highly "polluted" by the emission from the so-called bright grains (for more see the review by Rutten \& Uitenbroek 1991), which have a marked presence in the $\mathrm{Ca}$ II line but not in the $\mathrm{H} \alpha$ line. These bright grains are produced from shocks generated by vertically propagating acoustic waves that are just above the acoustic cut-off frequency, emanating from the photosphere (Carlsson \& Stein 1997). However, it should be noted that the downflows in the footpoints are well distinguishable in both lines; for details see the results of the Doppler velocities later in this section.

Next, we investigated the response of the chromospheric counterpart of the CBP to the magnetic-flux increase. First, we noticed an enhancement of the chromospheric activity associated with both the occurrence of HLs and mottles following the magnetic flux increase (see the animation in Fig. A.1). This is shown with an $\mathrm{H} \alpha$ light curve in the top panel of Fig. 5 produced from an area enclosing most of the CBP (R5 in Fig. 3). The light curve is obtained from the sum of the intensity in the blue and red wings of $\mathrm{H} \alpha$ at $\pm 0.45 \AA$, after subtracting and normalizing by the mean intensity. There is a clear decrease (enhanced presence of absorption features) with respect to the average intensity that indicates enhanced dynamics associated with new mottles and HLs. It should be noted that this activity appears to last longer (until 18:40 UT) than the heating event in the corona. As it could be seen later from the temperature analysis (Sect. 3.3.2), 

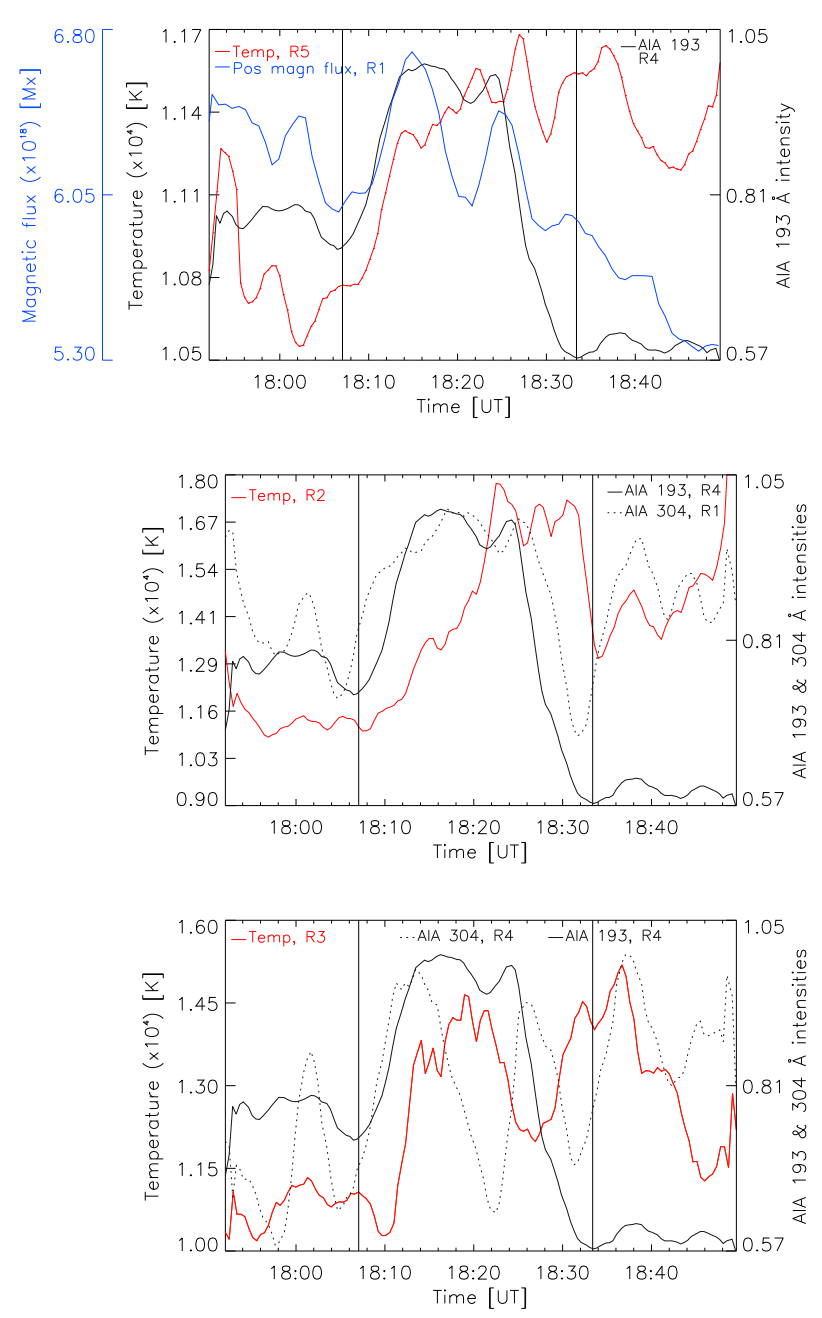

Fig. 7. Hydrogen temperature variations with respect to the magnetic flux, AIA 193, and AIA 304 intensity variations of the CBP. Top: averaged temperature obtained from the whole CBP (R5). The total positive magnetic flux (R1) and the AIA 193 intensity (R4). Middle: averaged temperature in the CBP south footpoint, R2. The AIA 304 is taken from the same area as the positive flux (R1). Bottom: temperature estimated from the small area of one of the HLs, R3. The AIA 304 is taken from the same area of the CBP loops as AIA 193, R4.

a hydrogen temperature enhancement also continues after the coronal response has ended. This is also visually noticeable from the middle and right panels of Fig. 3 and the provided animations in Figs. A.1 and A.2.

More detailed analysis was made for one of the HLs. In the middle and bottom panels of Fig. 5 we show the $\mathrm{H} \alpha$ linecore intensity and Doppler-shift temporal variations, respectively, obtained from a small $3 \times 3 \mathrm{px}^{2}$ area (R3, see the cross sign on the $\mathrm{H} \alpha$ images of Figs. 2 and 3 ) at one of the HLs. The reference profile was chosen as an average over the entire dataset from a region outside the CBP as shown in Fig. 2 (first panel in the third row, "Ref"). The $\mathrm{H} \alpha$ line-core intensity curve is produced by subtracting and normalizing by the mean $\mathrm{H} \alpha$ line-core intensity of this area. A signature of the $\mathrm{HL}$ is notable in the $\mathrm{H} \alpha$ curve after $\sim 18: 16$ UT (Fig. 5, middle panel). The HL disappears at $\sim 18: 28$ UT, which gives a life time of $\sim 12 \mathrm{~min}$. However, this life time should be taken with caution because it looks like the HL has moved horizontally, thus leaving the inspected location. Taking a larger area would have resulted in including another feature. As seen from the right panel in Fig. 3, the HL seems to be present for longer (i.e. $27 \mathrm{~min}$ ). After inspecting the animation, we concluded that a new upflow from the CBP footpoint along the same field line may possibly have led to the reappearance of this HL in the $\mathrm{H} \alpha$ images. The Doppler-shift pattern of the HL (Fig. 5, bottom panel) indicates upflows followed by downflows. It should be noted that as we investigated a location at almost the horizontal part of the loop, the Doppler values of the up- and downflows are very small. We inspected the animation sequences, which indicate that the $\mathrm{H} \alpha$ plasma in the HL seems to originate from upflows from the footpoints of the CBPs (see the animation in Fig. A.1).

We also investigated the general pattern of the $\mathrm{H} \alpha$ linecore intensity (produced after subtracting and normalizing by the mean $\mathrm{H} \alpha$ line-core intensity of this area) and Doppler shifts in the south footpoint of the CBP (Fig. 6, top and bottom panels, respectively). To accomplish this, we selected the same area as for the magnetic flux analysis (R1). The $\mathrm{H} \alpha$ line-core intensity curve indicates an intensity increase in the south footpoint, which begins together with the magnetic-flux increase. Almost 10 min after the start of the coronal heating period a distinct pattern of up- and downflows is apparent from the $\mathrm{H} \alpha$ Doppler-shift curve profile (Fig. 6, bottom panel), indicating increased chromospheric activity that is also discernible in the Doppler-shift curve of the Ca II line, although weaker. After 18:28 UT, a steep $\mathrm{H} \alpha$ line-core intensity decrease (Fig. 6, top panel) is associated with strong up and down cool-plasma flows that appear to be related to large mottle or surge-like events not related to the CBP phenomenon. The features propagate east and west of the CBP originating from the same polarity as the CBP south footpoint.

\subsubsection{Temperature evolution}

Important information is brought by the obtained hydrogen temperature at chromospheric levels which, as a reminder, was obtained with the multi-layer inversion technique of Chae et al. (2020), as explained in Sect. 2. In Fig. 7 we show the temperature temporal variation averaged over the whole CBP (R5 shown in Fig. 5, see also the animation in Fig. A.3). For reference we also show the AIA 193 and positive magnetic-flux temporal variations. The temperature of the whole CBP starts to rise steeply with a delay of less than $\sim 3 \mathrm{~min}$ (at $\sim 18: 10 \mathrm{UT}$ ) following the magnetic-flux and the coronal emission enhancements. The average temperature increase is $\sim 10 \%$ with respect to the pre-event temperature, from $1.07 \times 10^{4} \mathrm{~K}$ to $1.175 \times 10^{4} \mathrm{~K}$. The maximum value is reached $\sim 17 \mathrm{~min}$ later at 18:27 UT. The random temperature error in each pixel was estimated (Chae et al. 2020) at $140 \mathrm{~K}$. The chromospheric heating event lasts longer than the coronal heating event.

We also derived the averaged temperature from a small area of $11 \times 16 \mathrm{px}^{2}$ above the location of the positive flux in the south footpoint of the CBP indicated with a small black square on the HMI magnetograms in Fig. 2 (R2). Figure 7 (middle panel) shows the clear pattern of a significant temperature increase in the south footpoint of the CBP that start with a delay of less than $\sim 3$ min after the coronal intensity enhancement. The temperature initially increases reaching a small peak (at $1.35 \times 10^{4} \mathrm{~K}$ ) co-temporally with the magnetic flux and coronal intensity peaks. Initially, the increase is around $17 \%$, from $1.15 \times 10^{4} \mathrm{~K}$ at $\sim 18: 10 \mathrm{UT}$ to $1.35 \times 10^{4} \mathrm{~K}$ at $\sim 18: 15 \mathrm{UT}$. For the following $13 \mathrm{~min}$ the temperature sharply continues to rise, reaching $\sim 1.78 \times 10^{4} \mathrm{~K}$ at $\sim 18: 23 \mathrm{UT}$. Thus the increase with respect to the pre-event temperature is $55 \%$ (from $1.15 \times 10^{4} \mathrm{~K}$ to $\left.1.78 \times 10^{4} \mathrm{~K}\right)$. We believe that the longer duration of the temperature enhancement is related to the enhanced activity of 

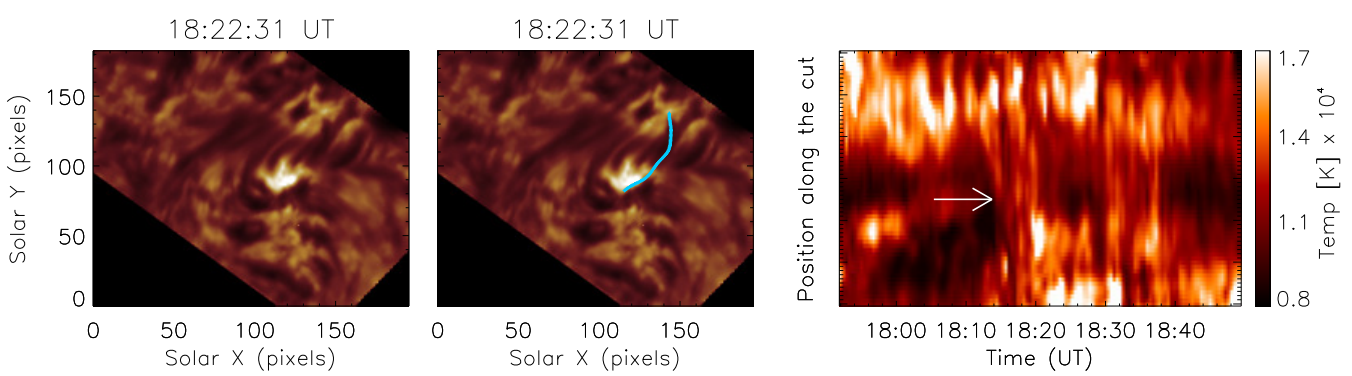

Fig. 8. Temperature increase in the HLs. Left and middle: $\mathrm{H} \alpha$ line-core intensity images. The blue line on the middle-panel image outlines the position of the pixels used to produce the hydrogen temperature time-distance image. Right: hydrogen temperature time-distance image. The arrow points at the start of the heating episode of the HLs. The pixel size is $0.16^{\prime \prime}$.

downflows, which is described Sect. 4. The temperature falls back sharply more than $\sim 20$ min later after the beginning of the coronal emission decrease has begun.

The temperature curve from one of the HLs (R3) was also produced and is shown in the bottom panel of Fig. 7. Again, the temperature increase in the HL location is evident. This increase starts with the appearance of the HL and lasts until the HL vanishes (see also the middle panel of Fig. 5). The temperature increase is $46 \%$, from $1.03 \times 10^{4} \mathrm{~K}$ to $1.5 \times 10^{4} \mathrm{~K}$. The maximum is reached during the re-appearance of the HL at 18:37 UT. The AIA 304 and the AIA 193 light curves both taken from the CBP loops show a co-temporal increase, while the emission in the AIA 304 (i.e. cooler transition-region loops) lasts a few minutes longer.

Furthermore, we demonstrate the CBP temperature increase with a time-slice image shown in Fig. 8. It was produced from the location of one of the HLs discussed above and traced in the $\mathrm{H} \alpha$ line-core image taken at 18:21:31 UT (shown in the left and middle panels in Fig. 8). This figure clearly shows the heating period of the HLs.

We should mention again (see Sect. 1) the study by Contarino et al. (2009) reporting on the morphological and dynamical evolution of an AFS observed in the Ca II 8542.1 line. We established that this AFS also represents a chromospheric counterpart of a CBP. The data were obtained in 2006 and therefore the only available coronal imaging data are from SoHO/EIT. The IBIS data were taken during the late stage of the CBP evolution called the decay phase. As in our study, in addition to the AFS rooted in a bipolar region, mottles showing upward and downward motions were also observed to evolve from these magnetic-flux concentrations. The study estimates the temperature of the AFS at $8.2 \times 10^{4} \mathrm{~K}$ in one footpoint and at about $2.4 \times 10^{4} \mathrm{~K}$ in the other, based on the Ca II Doppler width (see their Eq. (2)). These temperatures are higher than the footpoint values of $1.78 \times 10^{4} \mathrm{~K}$ obtained in the present study. The study also reports upflows in the body of the AFS and downflows in its footpoints during $7 \mathrm{~min}$ of observations (see their Table 2). The Doppler pattern is consistent with that obtained in this work during certain time periods. Our analysis identifies more complex evolution that may also be related to the magnetic-flux increase in our CBP.

\subsection{CBP magnetic topology}

\subsubsection{The overlying corona}

We also investigated the magnetic configuration of both the lowlying CBP loops and the overlying corona. Starting with the latter, we calculated a simple potential field extrapolation from the HMI magnetograms for four observed times, namely one at 17:21 UT, that is before the flux emergence and heating event described in the foregoing sections (Sect. 3.3), and three during the event, namely at 18:10 UT, 18:15 UT, and 18:25 UT. The calculated 3D structure was then searched for the presence of null points. To visualize and analyse the $3 \mathrm{D}$ magnetic field the Visualization and Analysis Platform for Ocean, atmosphere, and solar Researchers (VAPOR) software was used.

For the extrapolation, HMI data with a large FOV of $349.5^{\prime \prime} \times 349.5^{\prime \prime}$ were chosen as a basis. This is much larger than the area shown for the AIA 193 observations in Fig. 2 $\left(21^{\prime \prime} \times 39^{\prime \prime}\right)$, amounting to a total of $694 \mathrm{px} \times 694 \mathrm{px}$ with resolution $0.5^{\prime \prime} \mathrm{px}^{-1}$. The base area was essentially in flux balance, where the deviation between the total positive and negative flux is at the $2 \%$ level. To prevent boundary effects, before extrapolating the base data were apodized in a band of $40^{\prime \prime}$ at the rim, maintaining their average. We checked that the field-line geometry of the area of interest shown below is the same irrespective of the apodization.

Figure 9 illustrates the field-line structure above the CBP for the 17:21 UT observation. To facilitate the visualization, we made the choice of field lines giving extra weight to those that pass near to weak-field locations, so as to better illustrate the region around possible null points. We see that the field configuration is the fan-spine kind, in which the fan surface has an inclined dome shape that reaches down to the negative polarity at the surface (to help with the orientation: the string of negative elements towards the top-left of the top panel are the forebears of the set of negative elements in the top row in Fig. 2, which are for later times). Like in the simplest fan-spine configuration, the lower spine is rooted in one of the elements of the parasitic positive polarity. The parasitic polarity is distributed in a string of magnetic elements. The lower spine shown in the figure just attaches to one of them; the field lines issuing from the other elements are loops contained within the space below the fan surface. The upper spine, in turn, does not reach high in the corona but, instead, bends over and connects to positive elements outside of the CBP region to the right in the figure. In the bottom panel, we see that the CBP as detected by AIA 193 is located in a fraction of the dome only, similar to the situation described by Mou et al. (2018).

There remains the question of whether there is a null point at the junction of the spines with the fan surface. To search for this null point, we used the method developed by Haynes \& Parnell (2007). Through this method, we located a prominent null point exactly at that juncture, at a height of $6.8 \mathrm{Mm}$ above the surface. Comments to the structure and possible presence of null points for other observed times are deferred to the discussion. 


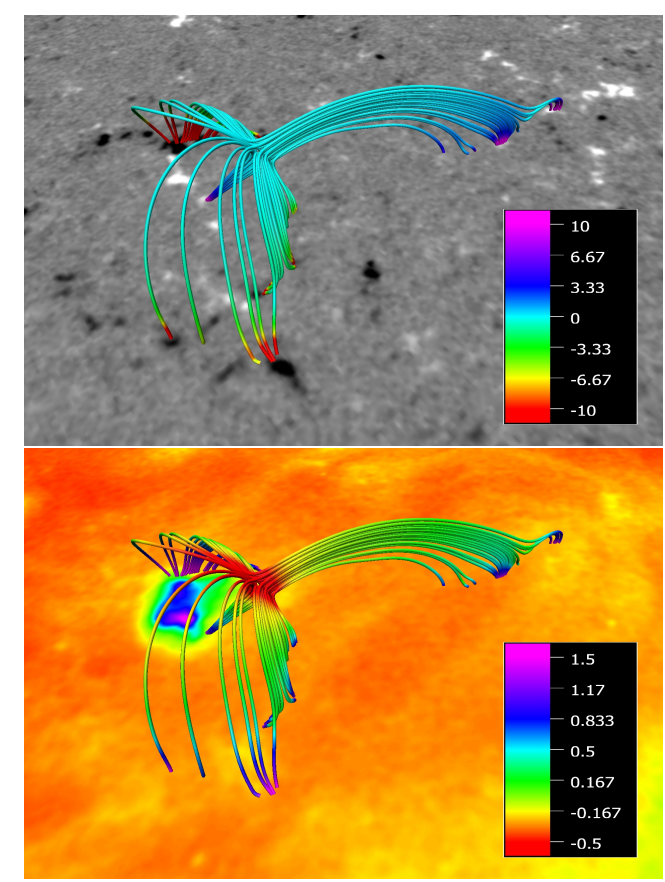

Fig. 9. Potential field extrapolation of the coronal magnetic field overlying the CBP at 17:21 UT. Top panel: background image is the HMI magnetogram from which the extrapolation has been made. The field lines are coloured according to the value of the vertical field component, with values in gauss as in the colour bar. Bottom panel: same but overlaid on a co-temporal AIA 193 image. The field line colours correspond to the logarithm of $|\boldsymbol{B}|$.

\subsubsection{Low-lying loops: LFFF extrapolation}

Next we investigated in detail the CBP magnetic field structures that confine plasmas emitting at coronal temperatures (see Sect. 4) using the LFFF method as explained in Sect. 2. We determinted the value of $\alpha \mathrm{L}$ by optimizing the match of the calculated loops to those apparent in the MGN-processed AIA 193 images. For the LFFF extrapolation we used the higher signalto-noise HMI 12 min cadence data. We started the loop identification with AIA 193 data taken as early as 16:00 UT, but failed to obtain any LFFF field lines that match the observed loops. At this time the CBP loops appeared strongly sigmoidal or curved, that is sheared, implying the presence of large free magnetic energy. Visual analysis of the AIA time series showe an ejection of cool material just before 16:00 UT, similar to the cases discussed in Mou et al. (2018). This clearly suggests that an eruptive phenomenon has taken place. As shown above (Fig. 1 and related discussion), a magnetic-flux increase started as early as 14:00 UT, which has possibly contributed to this activity. We were only able to identify some individual loops that could be fit with the LFFF model after 17:12 UT. In Figs. 10 and A.4 we give examples of two extrapolated loops and their parameters. We concentrated our analysis to only the largest loops because the small loops lie far too low in the solar atmosphere (below $1 \mathrm{Mm}$ ) and the extrapolation method becomes less reliable. Low-lying loops, including chromospheric loops, in the future will be modelled using the newly developed magneto-hydro-static extrapolation model (Zhu \& Wiegelmann 2019), which has higher accuracy for extrapolations at chromospheric heights. The analysis established that field lines with $\alpha \mathrm{L}$ of -3.0 and -3.5 best match the identified AIA 193 loops. During the analysed onehour observations the $\alpha \mathrm{L}$ parameter of the best-fitting field lines remained unchanged. The maximum loop heights were $\sim 3.5 \mathrm{Mm}$
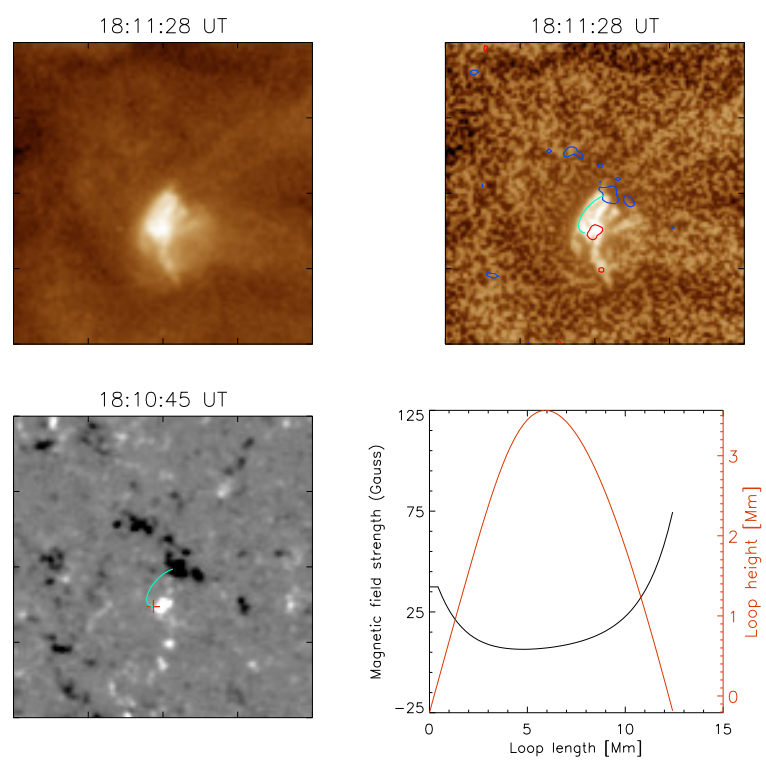

Fig. 10. Linear force-free field extrapolation of a CBP loop. Top left: AIA 193 image. Top right: MGN processed AIA 193 image with overplotted a magnetic field line that matches one of the extrapolated CBP loops. Bottom left: HMI magnetogram with the same magnetic field line. The cross indicates the location from which the magnetic field line was traced. Bottom right: magnetic-field-line parameters.

and lengths $\sim 12 \mathrm{Mm}$, respectively. Higher and longer field lines failed to align with the observed loops. The presented loops have magnetic field strengths of $75 \mathrm{G}$ (negative polarity) and 25 and $35 \mathrm{G}$ (positive polarity) at the photosphere, for the loops shown in Figs. 10 and A.4, respectively. As found by a study of Wiegelmann et al. (2010) and recently confirmed by Xie et al. (2017), the magnetic field strength is typically different in the two loop footpoints.

\section{Discussion}

The heating of plasmas confined in small-scale loops has been explored through numerous theoretical models (for a review see Sect. 9 in Madjarska 2019). A classic model for flux cancellation CBPs was proposed by Priest et al. (1994) (see also Parnell et al. 1994). This was a highly idealized, analytical, purely magnetic model of a CBP in two dimensions. This model starts with two unrelated opposite magnetic poles at the surface that move towards each other and considers a quasi-static approach, in which the field relaxes to a potential configuration continuously along the process. After reaching a critical mutual distance (the interaction distance), an X-type null point is created in the vertical axis between these two magnetic poles followed by reconnection at the X-type null point. The model was then tested numerically and extended by von Rekowski et al. (2006a,b) by solving the two-dimensional magnetohydrodynamics (MHD) equations for magnetic field and plasma in the isothermal, gravity-free approximation. Another fundamental approach using analytical theory, now in three dimensions, was made by Longcope (1998), who considered the approach of two magnetic poles at the surface that are nearing each other but not exactly along the line joining them. A 3D numerical model of this sort of flyby process was carried out by Galsgaard et al. (2000), who provided first glimpses of the complication of the $3 \mathrm{D}$ reconnection patterns that may be at the basis of some of the CBP structures. 
The recent model by Wyper et al. (2018) investigates the formation of CBPs in coronal holes. The study also explores the formation of associated collimated flows (i.e. jets), which is not the subject of the present study and is not discussed further. In this model the initial magnetic configuration corresponds to a fan-spine topology with a single 3D null-point and with the nullpoint spine axis connecting to the minority polarity flux. The structure was stressed by three types of surface flows. The first was a large-scale surface flow shearing only one part of the separatrix surface, the second represents a large-scale surface flow that shears part of the polarity inversion line around the minority flux, and for the third set-up the second shearing flow together with a flyby of the majority polarity flux that passes the moving minority polarity flux was employed. Small-loop structures (i.e. CBPs) with different morphologies were produced. The heating in these magnetic configurations was due to the shearing of the magnetic field near to the separatrix, which leads to steady interchange reconnection. This is coupled with quasi-periodic, lowintensity bursts of reconnection resulting in CBPs with periodically varying intensity. This model relies on a very high level of shear, far from the observational values, yet it shows that magnetic reconnection in the corona can produce a phenomenon that resembles a $\mathrm{CBP}$ with varying intensity.

The magnetic field-line configuration obtained in Sect. 3.4.1 for the overlying corona at 17:21 UT shows a clear fan-spine topology; the upper spine does not reach high coronal levels but, rather, bends over and connects to magnetic elements of positive polarity several tens of megameters away from the base of the CBP. By using the method of Haynes \& Parnell (2007), we also detected a clear null point at the junction between fan surface and spines. Interestingly, the projection of the AIA 193 CBP observation fits very well in the area between the root of the lower spine in the parasitic positive polarity and the comparatively strong negative magnetic elements at the intersection of the fan surface with the photosphere on the northern side of the complex. This one-sidedness for the CBP with respect to the fan-spine configuration is also apparent in most of the field configurations for CBPs calculated by Galsgaard et al. (2017). It is precisely in that domain that the flux emergence and heating event described in detail in Sect. 3.3 takes place. That heating event occurred some $50 \mathrm{~min}$ after the 17:21 UT observation. To complete the coronal view, therefore, we also calculated potential field extrapolations for three times at the beginning (18:10 UT), in the middle (18:15 UT), and towards the end (18:25 UT) of said event. We obtained field-line configurations for all three times, which are very similar in their general fan-spine structure to that of the earlier time, albeit with the possibly important difference that for the first two, those at 18:10 UT and 18:15 UT, no null point could be detected. Those two times coincide with the rising period of the magnetic flux at the surface due to the flux emergence episode.

As already mentioned above, during the analysed observational period of only $\sim 1 \mathrm{~h}$, restricted by the ground-based observations, we identified a short period of photospheric magnetic flux increase in the footpoints of the CBP related to flux emergence. Aftereffects of the magnetic-flux increase are observed in both the coronal and chromospheric components of the CBP. We detected a co-temporal rise of the coronal emission, indicating that the CBP loops are heated to coronal temperatures. At the time of this coronal emission enhancement the magnetic field concentrations where the footpoint of the CBP are rooted do not show any significant displacement or convergence. Therefore, if the heating has occurred at coronal heights caused by magnetic reconnection, the driver is possibly related to random small-scale footpoint motions that are visually not possible to follow in the observations. If magnetic reconnection has taken place in the corona, thermal conduction, which efficiently transports heat along the magnetic field lines at temperatures above $\log T(\mathrm{~K}) \geq 4.7$, would uniformly distribute the heating along the CBP loops. This would be detected through the coronal observations by an emission enhancement in the coronal channels of AIA, for example AIA 193. The reconnection process must be relatively steady as no signature of bursty emission is detected in the coronal loops. Another possible explanation is that the reconnection occurred on spatial scales far smaller than the present instrumentation can spatially resolve, thus we detect only the aftereffects, namely the uniformly heated coronal loops along their entire length.

A heating episode is also detected in the chromospheric counterpart of the CBP observed in the $\mathrm{H} \alpha$ line. We make a small diversion here to discuss the chromospheric morphology of the CBP. In the $\mathrm{H} \alpha$ line-core intensity images the morphology of the $\mathrm{CPB}$ is seen as a bundle of elongated dark features that have a sigmoidal shape at the one end (south). As it has been shown by Leenaarts \& Carlsson (2012) from their 3D radiation-MHD simulations combined with three-dimensional non-LTE radiative transfer computations of the $\mathrm{H} \alpha$ line, dark elongated structures seen in the $\mathrm{H} \alpha$ line core, referred to as fibrils, trace magnetic field lines to a large extent. Their fibrils were explicitly found within $\beta=0.01$ contours, following the magnetic field direction between opposite flux concentrations. In the case of our CBP, these features connect the CBP bipoles, and therefore represent the chromospheric counterpart of the CBP. Thus, the term we adopted, HLs, best describes the observed features. The HLs and coronal loops appear to be separate entities with magnetic-field structures rooted in different elements of the same magnetic-flux concentrations. As already suggested by several studies, CBPs appear to be composed of loops at different temperatures (e.g., Habbal et al. 1990; Doschek et al. 2010; Kwon et al. 2012). The interconnection between coronal and lower-temperature loops and its temporal evolution has never been explored, possibly because of a lack of suitable observations. To identify the precise rooting point of these two different loop systems requires highresolution vector magnetic-field observations and extrapolation models that can successfully cope with both coronal and chromospheric fields. Such a model is, as mentioned above, presently under development (Zhu \& Wiegelmann 2019), and it will be employed in the future when suitable vector magnetograms are obtained. The HMI vector magnetograms are insufficient owing to the low signal-to-noise of the transverse component of the field in the quiet Sun (Hoeksema et al. 2014).

During the coronal heating episode, the chromospheric counterpart of the CBP exhibits a rapid (hydrogen) temperature increase; specific locations such as a CBP footpoint and a $\mathrm{H} \alpha$ loop show a temperature rise of in average $\sim 50 \%$ with respect to the pre-event value. Also, following the magnetic flux and temperature increase in the corona, the chromospheric counterpart of the CBP becomes more dynamic for an interval of about $30 \mathrm{~min}$, which is longer than the duration of the coronal loop heating. The typically observed downflows are enhanced possibly by plasma draining from chromospheric, transition-region, and coronal heights. However, upflows, detected as mottles and plasma motions along the CBP loops, also appear to intensify after the magnetic-flux emergence. Both the hydrogen temperature and the dynamic flows appear to occur with a small delay of less than 3 min after the coronal heating episode has started. This delay together with the identified coronal field structure suggest the scenario of reconnection-associated coronal heating. To the best of our knowledge, the only other study that has looked into 
and reported such a delay is the paper by Habbal \& Withbroe (1981) from the Skylab spectroheliogram observations of nine CPBs. The study found a delay of at least $5.5 \mathrm{~min}$ (the cadence of their data) between the response in the coronal $\mathrm{Mg}$ X $625 \AA$, and transition-region O VI $1032 \AA$, C II $1335 \AA$, and the chromospheric Ly- $\alpha 1216 \AA$ lines.

There are several possible sources for the temperature increase in the chromosphere underlying the CBP loops. The combined effect of the thermal conduction and energetic particle beams would result in a temperature increase in the coronal loop footpoints. Below temperatures of $\log T(\mathrm{~K}) \geq 4.7$, the thermal conduction would be less effective and heating by energetic particle beams would prevail. Magnetic reconnection in the corona is known to generate beams of accelerated particles that are typically observed during solar flares and are known to be responsible for the heating in the chromospheric footpoints of coronal loops. However, a very recent study by Frogner et al. (2020) has demonstrated that particle beams can also be a common phenomenon in the quiet Sun and would largely contribute to the energy transport from the corona into the chromosphere. Another contributor to the footpoint temperature increase would be shocks caused by the downflows as typically observed in sunspot plumes (footpoints of large extended loops rooted in sunspot umbras) for instance (e.g., Kleint et al. 2014). In addition, the enthalpy flux due to downflows overwhelms the conductive flux in the low transition region and below, thereby triggering the loop leg or footpoint temperature increase (Chae et al. 1997).

We ask how the heating in the coronal loops and their foopoints in the chromosphere could lead to a heating of the chromospheric loops. A plausible mechanism is through radiative heating, in which the Ly- $\alpha$ line is the main source together with heating from EUV optically thin radiative losses from the transition region and corona absorbed in the chromosphere (for details see Carlsson \& Leenaarts 2012; Zacharias et al. 2018). To confirm this further numerical simulations are required. A delayed intense increase of the Ly- $\alpha 1216 \AA$ emission was reported by Habbal \& Withbroe (1981) during a heating episode initially recorded in the $\operatorname{Mg}$ X $625 \AA$ line.

We should also mention that enhanced nanoflare heating due to magnetic flux increase could be another possible heating mechanism. This heating mechanism has already been explored (see Sect. 9 in Madjarska 2019), but the result remains inconclusive and requires further observational evidence. Unfortunately, our study cannot provide observational evidence for this mechanism because this would require spectroscopic observations covering a wide range of formation temperatures combined with nanoflare heating modelling.

\section{Summary and conclusions}

The present study has investigated, for the first time since the work by Habbal \& Withbroe (1981), the temporal variations of the coronal and chromospheric emission from CBP together with the temporal changes of the magnetic flux in the footpoints of a CBP. Ground-based $\mathrm{H} \alpha$ and $\mathrm{Ca}$ II data are used to trace changes of both intensities and Doppler velocities, and most importantly, of the hydrogen temperature based on a novel, recently developed inversion technique by Chae et al. (2020). The observed coronal heating caused by magnetic flux emergence is followed with a small delay by enhanced dynamics and heating of the chromospheric loops and footpoints the CBP. The delay in the response of the chromospheric counterpart of the CBP could suggest that the heating occurs at coronal heights with a conse- quent heating of the CBP chromospheric counterpart. An additional heating generated in the chromosphere, from magnetic reconnection for example, may also be at work but this case study does not provide evidence for this. This scenario will be explored in the future through new CBP multi-temperature observations from ground- and space-based observatories and 3D radiation-MHD Bifrost simulations (Gudiksen et al. 2011).

Acknowledgements. We would like to thank very much the anonymous referee for their very helpful suggestions. This work was supported by the National Research Foundation of the Korea (NRF-2019H1D3A2A01099143, NRF-2020R1A2C2004616). FMI is grateful to the Spanish Ministry of Science, Innovation and Universities for support through projects AYA201455078-P and PGC2018-095832-B-I00; FMI and DNS gratefully acknowledge the European Research Council (ERC) for the award of the Synergy Grant "The Whole Sun" (ERC-2018-SyG 810218). DNS thankfully acknowledges support from the Research Council of Norway through its Centres of Excellence scheme, project number 262622. VAPOR is a product of the National Center for Atmospheric Research's Computational and Information Systems Lab. Support for VAPOR is provided by the US National Science Foundation (grants \# 03-25934 and 09-06379, ACI-14-40412), and by the Korea Institute of Science and Technology Information. The HMI data are provided courtesy of NASA/SDO and corresponding science teams. The HMI data have been retrieved using the Stanford University's Joint Science Operations Centre/Science Data Processing Facility. We thank Thomas Wiegelmann for providing the LFFF code. The authors thank the ISSI (Bern) for the support to the team "Observation-Driven Modelling of Solar Phenomena".

\section{References}

Andretta, V., Telloni, D., \& Del Zanna, G. 2012, Sol. Phys., 279, 53

Carlsson, M., \& Leenaarts, J. 2012, A\&A, 539, A39

Carlsson, M., \& Stein, R. F. 1997, ApJ, 481, 500

Cauzzi, G., Reardon, K. P., Uitenbroek, H., et al. 2008, A\&A, 480, 515

Chae, J., Yun, H. S., \& Poland, A. I. 1997, ApJ, 480, 817

Chae, J., Park, H.-M., Ahn, K., et al. 2013, Sol. Phys., 288, 1

Chae, J., Yang, H., Park, H., et al. 2014, ApJ, 789, 108

Chae, J., Madjarska, M. S., Kwak, H., \& Cho, K. 2020, A\&A, 640, A45

Cheung, M. C. M., \& Isobe, H. 2014, Liv. Rev. Sol. Phys., 11, 3

Contarino, L., Zuccarello, F., Romano, P., Spadaro, D., \& Ermolli, I. 2009, A\&A, 507,1625

Dacie, S., Török, T., Démoulin, P., et al. 2018, ApJ, 862, 117

Doschek, G. A., Landi, E., Warren, H. P., \& Harra, L. K. 2010, ApJ, 710, 1806

Frogner, L., Gudiksen, B. V., \& Bakke, H. 2020, A\&A, 643, A27

Galsgaard, K., Parnell, C. E., \& Blaizot, J. 2000, A\&A, 362, 395

Galsgaard, K., Madjarska, M. S., Moreno-Insertis, F., Huang, Z., \& Wiegelmann, T. 2017, A\&A, 606, A46

Golding, T. P., Leenaarts, J., \& Carlsson, M. 2017, A\&A, 597, A102

Gudiksen, B. V., Carlsson, M., Hansteen, V. H., et al. 2011, A\&A, 531, A154

Habbal, S. R., \& Withbroe, G. L. 1981, Sol. Phys., 69, 77

Habbal, S. R., Withbroe, G. L., \& Dowdy, J. F., Jr. 1990, ApJ, 352, 333

Hansteen, V., Ortiz, A., Archontis, V., et al. 2019, A\&A, 626, A33

Haynes, A. L., \& Parnell, C. E. 2007, Phys. Plasmas, 14, 082107

Hoeksema, J. T., Liu, Y., Hayashi, K., et al. 2014, Sol. Phys., 289, 3483

Kayshap, P., \& Dwivedi, B. N. 2017, Sol. Phys., 292, 108

Kleint, L., Antolin, P., Tian, H., et al. 2014, ApJ, 789, L42

Kwon, R.-Y., Chae, J., Davila, J. M., et al. 2012, ApJ, 757, 167

Leenaarts, J., Carlsson, M., \& Rouppe van der Voort, L. 2012, ApJ, 749, 136

Lemen, J. R., Title, A. M., Akin, D. J., et al. 2012, Sol. Phys., 275, 17

Longcope, D. W. 1998, ApJ, 507, 433

Madjarska, M. S. 2019, Liv. Rev. Sol. Phys., 16, 2

Madjarska, M. S., Doyle, J. G., Teriaca, L., \& Banerjee, D. 2003, A\&A, 398, 775

Madjarska, M. S., Galsgaard, K., Mackay, D. H., Koleva, K., \& Dechev, M. 2020, A\&A, 643, A19

Morgan, H., \& Druckmüller, M. 2014, Sol. Phys., 289, 2945

Mou, C., Madjarska, M. S., Galsgaard, K., \& Xia, L. 2018, A\&A, 619, A55

Nóbrega-Siverio, D., Martínez-Sykora, J., Moreno-Insertis, F., \& Rouppe van der Voort, L. 2017, ApJ, 850, 153

O’Dwyer, B., Del Zanna, G., Mason, H. E., Weber, M. A., \& Tripathi, D. 2010, A\&A, 521, A21

Parnell, C. E., Priest, E. R., \& Golub, L. 1994, Sol. Phys., 151, 57

Pesnell, W. D., Thompson, B. J., \& Chamberlin, P. C. 2012, Sol. Phys., 275, 3

Priest, E. R., Parnell, C. E., \& Martin, S. F. 1994, ApJ, 427, 459

Rutten, R. J. 1999, ASP Conf. Ser., 184, 181

Rutten, R. J., \& Uitenbroek, H. 1991, Sol. Phys., 134, 15 
Scherrer, P. H., Schou, J., Bush, R. I., et al. 2012, Sol. Phys., 275, 207

Tian, H., Curdt, W., Marsch, E., \& He, J. 2008, ApJ, 681, L121

Tsiropoula, G., Tziotziou, K., Kontogiannis, I., et al. 2012, Space Sci. Rev., 169, 181

von Rekowski, B., Parnell, C. E., \& Priest, E. R. 2006a, MNRAS, 366, 125

von Rekowski, B., Parnell, C. E., \& Priest, E. R. 2006b, MNRAS, 369, 43

Wiegelmann, T., \& Neukirch, T. 2002, Sol. Phys., 208, 233

Wiegelmann, T., Solanki, S. K., Borrero, J. M., et al. 2010, ApJ, 723, L185

Wyper, P. F., DeVore, C. R., Karpen, J. T., Antiochos, S. K., \& Yeates, A. R. 2018, ApJ, 864, 165

Xia, L. D., Marsch, E. \& Curdt, W. 2003, A\&A, 399, L5

Xie, H., Madjarska, M. S., Li, B., et al. 2017, ApJ, 842, 38

Zacharias, P., Hansteen, V. H., Leenaarts, J., Carlsson, M., \& Gudiksen, B. V. 2018, A\&A, 614, A110

Zhu, X., \& Wiegelmann, T. 2019, A\&A, 631, A162

\section{Appendix A: Additional figures}

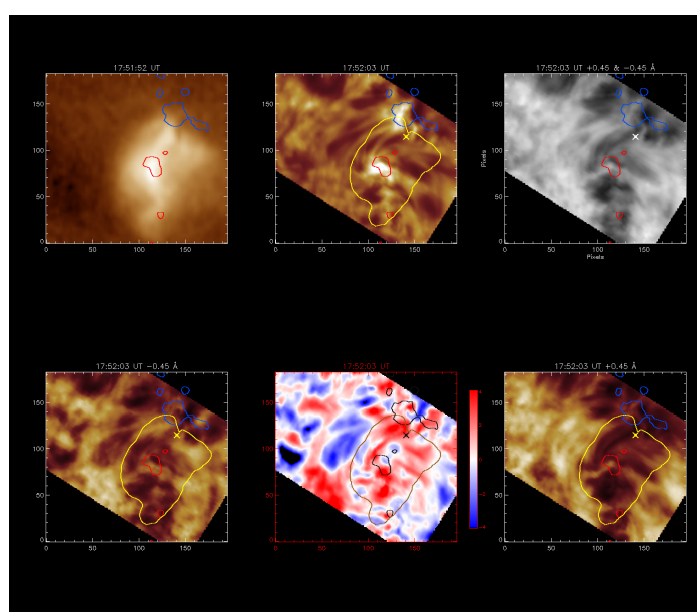

Fig. A.1. Animation of the following image sequences. From left to right in the top row: AIA 193 image, $\mathrm{H} \alpha$ line-core image, and $\mathrm{H} \alpha$ image produced from the sum of the intensity in the blue and red wing of $\mathrm{H} \alpha$ at \pm 45 Å. Bottom row: $\mathrm{H} \alpha$ image at $-0.45 \AA$, Doppler-shift image, and $\mathrm{H} \alpha$ image at $0.45 \AA$. The contour is the CBP AIA 193 intensity contour. The $\pm 50 \mathrm{G}$ magnetic-field contours (red-positive polarity; blue - negative). See online movie.

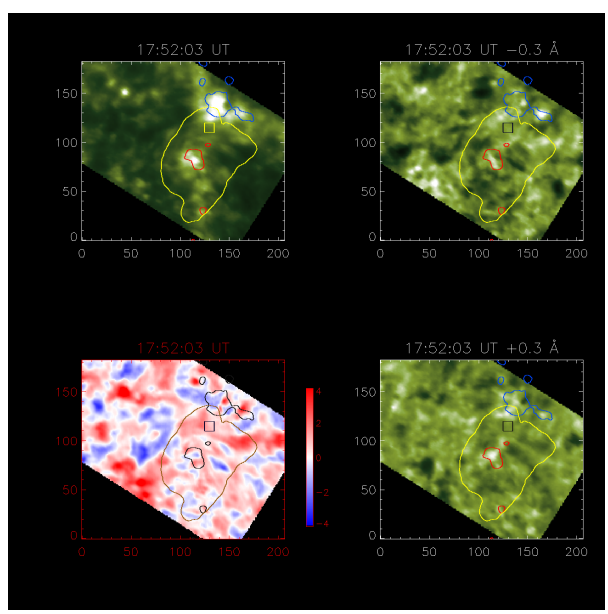

Fig. A.2. Animation of the following image sequences. Top row: Ca II line-core intensity image (left) and Ca II image at $-0.3 \AA$ (right). Bottom row: Doppler-shift image and Ca II image at $0.3 \AA$. The contour overplotted on all panels is the CBP AIA 193 intensity contour. The $\pm 50 \mathrm{G}$ magnetic-field contours (red - positive polarity; blue - negative). See online movie.

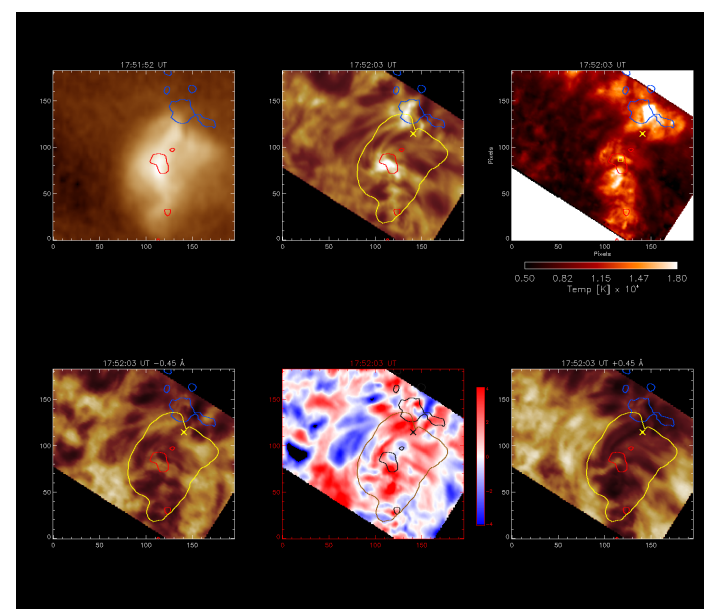

Fig. A.3. Animation of the following image sequences. From left to right in the top row: AIA 193 image, $\mathrm{H} \alpha$ line-core image, and hydrogen temperature image. Bottom row: $\mathrm{H} \alpha$ image at $-0.45 \AA$, Doppler-shift image, and $\mathrm{H} \alpha$ image at $+0.45 \AA$. The contour is the CBP AIA 193 intensity contour. The $\pm 50 \mathrm{G}$ magnetic-field contours (red - positive polarity; blue - negative). See online movie.
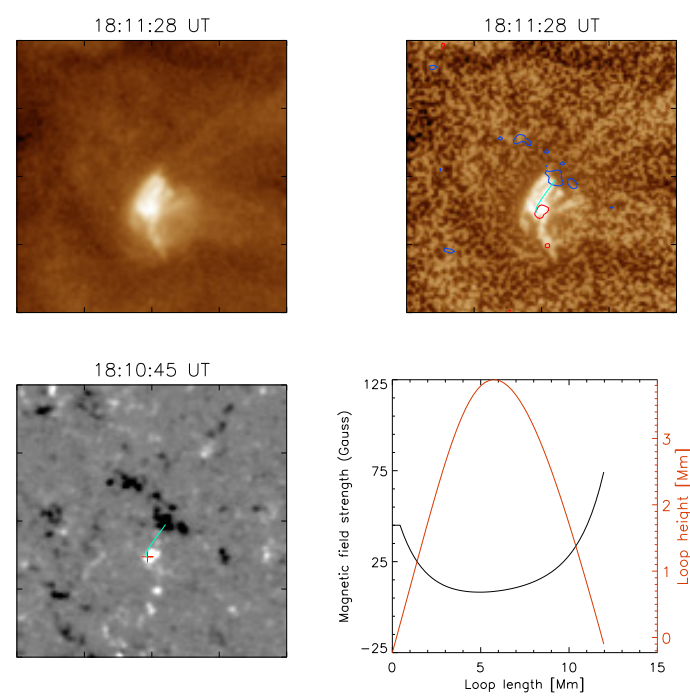

Fig. A.4. Linear force-free field extrapolation of a CBP loop. Top left: AIA 193 image. Top right: MGN processed AIA 193 image overplotted with a magnetic field line that matches one of the extrapolated CBP loops. Bottom left: HMI magnetogram overplotted with the same magnetic field line. The cross indicates the location from which the magnetic-field line was traced. Bottom right: magnetic-field-line parameters. 